\title{
Overview
}

\section{Combination of Novel Agents with Radiotherapy to Treat Rectal Cancer}

\author{
T.A. Greenhalgh, C. Dearman, R.A. Sharma \\ NIHR Oxford Biomedical Research Centre, CRUK-MRC Institute for Radiation Oncology, Department of Oncology, University of \\ Oxford, Oxford, UK
}

Received 9 August 2015; received in revised form 25 October 2015; accepted 26 October 2015

\begin{abstract}
Neoadjuvant chemoradiotherapy with fluoropyrimidines is an established treatment in the management of locally advanced rectal cancer. There has been a great deal of research into improving patient outcomes by modifying this regimen by the addition of further radiosensitising agents. One of the difficulties in advancing new combination therapies has been lack of consensus on which surrogate measures best reflect clinically important outcomes. Here we review combinations of the cytotoxic, biological and other agents currently under scrutiny to improve clinical outcomes for patients with colorectal cancer. We also discuss advances in biomarkers that may ultimately result in an ability to tailor neoadjuvant chemoradiotherapy regimens to the somatic gene profile of individual patients.
\end{abstract}

(c) 2015 The Royal College of Radiologists. Published by Elsevier Ltd. All rights reserved.

\section{Statement of Search Strategies Used and Sources of Information}

Data for this review article were identified through a search of Embase, Medline and Web of Science. The following terms were used together with any derivatives: colorectal cancer, radiosensitiser, radiotherapy, radiation, chemoradiotherapy, chemotherapy, drug therapy, novel agent, targeted agent, biological agent, bevacizumab, aflibercept, cetuximab and panitumumab. Only articles published in English were selected. The search also included the reference list for these articles and selected additional articles judged to be relevant.

\section{Introduction}

Although the original demonstration of significant radiosensitisation by a chemotherapeutic agent in combination

Author for correspondence: T.A. Greenhalgh, Department of Oncology, University of Oxford, Old Road Campus Research Building, Roosevelt Drive, Oxford OX3 7DQ UK. Tel: +44-1865-617330.

E-mail address: tessa.greenhalgh@oncology.ox.ac.uk (T.A. Greenhalgh). with radiotherapy for rectal cancer was in the adjuvant setting [1], clinical practice has moved away from adjuvant radiotherapy to the current practice of neoadjuvant chemoradiotherapy (CRT) [2,3]. The combination of neoadjuvant radiotherapy with a fluoropyrimidine is now an accepted standard of care in the treatment of locally advanced rectal cancer [4].

Significant debate exists regarding the best primary end points for clinical trials testing the addition of a new radiosensitising agent to CRT. Pathological complete response (pCR), the absence of viable tumour cells within the resection specimen, is commonly used but evidence is indeterminate as to whether this translates into improved outcome in terms of overall survival and disease-free survival (DFS; the time from randomisation until local or distant disease recurrence, or death) [5]. Other potential surrogate outcomes include downstaging rate, R0 resection (complete resection with no residual disease at margin) or circumferential resection margin (CRM: the minimum distance between the nearest extent of the tumour and the resection margin). Failure to achieve a negative CRM is associated with a high risk of local recurrence, but it is unclear as to whether this reflects inadequate surgery or aggressive disease [5]. DFS has been found to correlate with overall 
survival in a meta-analysis of colon cancer adjuvant trials [6] and at present is considered the most meaningful primary end point in phase III randomised control trials (RCTs) of CRT, albeit with the need to control for adjuvant chemotherapy. DFS is being used as the primary end point in the ARISTOTLE phase III trial in the UK, which is assessing the addition of irinotecan to CRT for rectal cancer. On account of the controversy over the best end points to use in clinical studies, here we will detail the various clinical outcomes reported in neoadjuvant CRT trials.

\section{Chemotherapy}

\section{Irinotecan}

Pre-clinical data have suggested that the radiosensitising properties of camptothecin derivatives may relate to the inhibition of potentially lethal damage repair. Irinotecan stabilises topoisomerase-I, an intranuclear enzyme that relaxes supercoiled DNA, by introducing a single-strand break through which the intact strand passes prior to religation. Collision between the irinotecan-topoisomerase I complex and the replication fork results in the formation of double-strand breaks, leading to G2 phase cell cycle arrest and cell death $[7,8]$.

Several early phase trials have assessed the addition of irinotecan to standard CRT with fluoropyrimidines for rectal cancer. Table 1 includes results from larger, published phase II trials, in which pCR rates varied from 13.7 to $37 \%$. An abstract by Jung et al. [17] details one of two randomised trials of CRT \pm irinotecan. With 142 participants, a pCR rate of $17.2 \%$ was achieved in the arm receiving 5-fluorouracil/ leucovorin (5-FU/LV) CRT versus $24.2 \%$ with a combination of $\mathrm{S} 1$, irinotecan and radiotherapy $(P=0.1)$. A significantly higher response rate was found in the latter arm when combining those achieving complete and near complete response (57.6\% versus $39.1 \%, P=0.035)$.

In a RCT of 5-FU with hyperfractionated radiotherapy versus 5-FU, irinotecan and $45 \mathrm{~Gy} / 25$ fractions with boost, Mohiuddin and colleagues [11] showed a pCR rate of $26 \%$ in both arms with no difference in rates of tumour downstaging. However, there was a higher proportion of radiotherapy delays in the irinotecan arm, 45\% versus $22 \%$. Overall, grade 3-4 toxicity was 51\% with irinotecan and $42 \%$ without the additional drug; gastrointestinal effects were most common in both arms (37\% versus 28\%) [11]. These rates were higher than those seen in single-arm trials (Table 1) where radiotherapy and chemotherapy dose intensity was largely maintained. Mohiuddin et al. [11] reported late toxicity rates of $6 \%$, lower than expected and again gastrointestinal effects predominated.

Five year outcomes have been published by Yoon et al. [15] and Mohiuddin et al. [19]. The latter study revealed overall survival 61\% (95\% confidence interval 47-74\%) versus 75\% (95\% confidence interval 61-85\%), distant failure $16 \% / 21 \%$ and locoregional failure (LRF) $16 \% / 17 \%$ rates without and with irinotecan, respectively. By comparison, in a review of 115 patients who had undergone a regime of
CRT with irinotecan/S1 in phase I/II trials, the overall pCR rate was similar at $24 \%$, with 5 year overall survival higher at $87 \%$, DFS 79\%, distant failure 17\% and LRF 2.6\% [20]. The multicentre UK-based phase III ARISTOTLE trial, recruiting since 2011, aims to confirm the potential improvement in outcomes seen with the addition of irinotecan to CRT and is currently set to complete recruitment in autumn 2016 [21].

\section{Oxaliplatin}

Oxaliplatin acts as a radiosensitiser through a variety of mechanisms, including causing DNA damage through the formation of inter- and intra-strand cross-links, induction of G2/M cell cycle arrest and blockade of DNA repair [22,23].

The addition of oxaliplatin to neoadjuvant CRT regimens showed promise in early phase single-arm trials, with pCR rates from $13 \%$ [24] to over $20 \%$ in several trials [25-27]. Five large phase III RCTs have gone on to compare various fluoropyrimidine-based neoadjuvant CRT regimens with or without oxaliplatin (Table 2) with pCR rates between 13.3 and $19.5 \%$ in the experimental arm compared with 11.3-17.8\% without oxaliplatin. CAO/ARO/AIO-04 was the only trial to show a significant difference between the two arms, with pCR rates of $13 \%$ in the control arm versus $17 \%$ with oxaliplatin $(P=0.038)$. The ACCORD trial found a difference of $13.9 \%$ versus $19.2 \%(P=0.09)$ but was powered to detect an increase from $11 \%$ to $20 \%$ with CAPOX. Of note, a higher dose of radiotherapy was given in the arm receiving oxaliplatin, which makes the results difficult to interpret.

A meta-analysis carried out by An et al. in 2013 [37], including results from ACCORD, AIO-04, NSABP R-04 and STAR-01 trials, did favour CRT with oxaliplatin (odds ratio $=1.20 ; 95 \%$ confidence interval $1.01-1.42 ; P=0.04$ ) with an absolute pCR rate difference of $2.5 \%$. However, PETACC-6 was not included in this analysis and with over 1000 participants showed no significant difference in $\mathrm{PCR}$, $11.3 \%$ without versus $13.3 \%$ with oxaliplatin $(P=0.31)$. Downstaging rates were also similar at $43.5 \%$ versus $41.5 \%$, higher than reported in NSAPB R-04 (23.5\% versus 17.9\%; $P=0.2$ ).

In terms of survival outcomes, recently published results from CAO/ARO/AIO-04 [32] showed a significant increase in 3 year DFS in the investigational group of $75.9 \%$ versus $71.2 \%$ in the control group (hazard ratio 0.79 ; $95 \%$ confidence interval $0.64-0.98 ; P=0.03$ ). Of note, the former received oxaliplatin with both CRT and adjuvant therapy, with the control group receiving 5-FU alone. Although PETACC-6 also added oxaliplatin to neoadjuvant and adjuvant regimens in experimental arm B, interim 3 year results indicated no significant improvement in DFS, being 73.9\% (95\% confidence interval $69.5-77.8 \%$ ) versus $74.5 \%$ (95\% confidence interval 70.1-78.3\%) in arm A, higher than anticipated. Follow-up is ongoing, but these results appear similar to the NSABP R-04 and ACCORD trials, which did not specify adjuvant chemotherapy regimens and reported no significant differences in DFS at 5 and 3 years, respectively.

Although in ACCORD, Dworak TRG score (hazard ratio 0.68 ; $95 \%$ confidence interval $0.59-0.79$ ) was found to be significantly correlated with 3 year DFS on multivariate 
Table 1

Phase II trials of neoadjuvant chemoradiotherapy (CRT) with fluoropyrimidine and irinotecan

\begin{tabular}{|c|c|c|c|c|c|c|c|c|c|}
\hline Refer & Pha & Stage* & $\begin{array}{l}\text { Systemic treatment } \\
\left(\mathrm{mg} / \mathrm{m}^{2}\right)\end{array}$ & RT (Gy/fractions) $\dagger$ & $n$ & $\mathrm{pCR}$ & Other end points & $\begin{array}{l}\text { Grade } 3-4 \text { toxicity } \\
\text { (non-surgical) }\end{array}$ & $\begin{array}{l}\text { Surgical } \\
\text { outcomes }\end{array}$ \\
\hline [9] & $\mathrm{I} / \mathrm{II}$ & Unresectable & $\begin{array}{l}\text { 5-FU 200/day } \\
\text { Irino } 60 \text { days } 1,8,15,22 \\
\text { ADJ: not defined }\end{array}$ & $45 / 25$ & 31 & $19 \%$ & $\begin{array}{l}\text { cCR } 29 \% \\
\text { Microfocal residual 15\% } \\
\text { Irino } 97 \% \text { had } 4 \text { cycles } \\
5 \text {-FU } 10 \% \text { DR } \\
\text { Median follow-up } 24 \\
\text { months: } 16 \% \text { LRF, } 26 \% \text { DF, } \\
\text { 10\% mortality }\end{array}$ & $\begin{array}{l}\text { Grade } 3 \text { : diarrhoea } 13 \% \text {, } \\
\text { constipation } 6 \% \text {, skin } \\
\text { sores } 3 \% \text {, lethargy } 3 \% \text {, } \\
\text { infection } 3 \% \text {, abdominal } \\
\text { cramping } 3 \% \text {, chest pain } \\
3 \% \\
\text { Grade } 4 \text { : fever } 3 \%\end{array}$ & $\begin{array}{l}90 \% \text { resected, } \\
81 \% \text { R0 } \\
1 \text { death }(A L)\end{array}$ \\
\hline [10] & II & $\mathrm{uT} 3 / 4$ or $\mathrm{N}+$ & $\begin{array}{l}\text { 5-FU } 200 / \text { day } \\
\text { Irino } 50 \text { days } 1,8,15,22 \\
\text { ADJ: Up to } 4 \text { months } 5 \\
\text {-FU/LV }\end{array}$ & $50.4 / 28$ & 32 & $37 \%$ & $\begin{array}{l}\text { DS } 34 \% \\
5-F U \text { DR } 32 \% \\
\text { Irino DR } 32 \% \\
6 \% \text { metastases on restaging }\end{array}$ & $\begin{array}{l}\text { Grade } 3 \text { : diarrhoea } 28 \% \text {, } \\
\text { mucositis } 21 \% \text {, skin sores } \\
21 \% \text {, abdominal cramps } \\
9 \% \text {, N\&V } 3 \%\end{array}$ & $\begin{array}{l}100 \% \text { resected, } \\
50 \% \text { SSS } \\
\text { AL } 3 \%\end{array}$ \\
\hline [11] & II & $\begin{array}{l}\text { cT3 } / 4, \leq 9 \mathrm{~cm} \text { from } \\
\text { dentate line }\end{array}$ & $\begin{array}{l}\text { A: 5-FU 225/day } \\
\text { B: 5-FU } 5 \text { days/ } \\
\text { week + Irino 50/week } \\
\times 4 \\
\text { ADJ: Recommended if } \\
\text { pathologic residual } \\
\text { disease }\end{array}$ & $\begin{array}{l}\text { A: } 45.6 / 38 \\
\text { BD }(\geq 6 \mathrm{~h} \text { apart }) \\
+ \text { boost } 9.6(\mathrm{~T} 3) 14.4(\mathrm{~T} 4) \\
\text { B: } 45 / 25 \\
+ \text { boost } 5.4(\mathrm{~T} 3) 9(\mathrm{~T} 4)\end{array}$ & $\begin{array}{l}\text { A: } 50 \\
\text { B: } 53\end{array}$ & $\begin{array}{l}\text { A: } 26 \% \\
\text { B: } 26 \%\end{array}$ & $\begin{array}{l}\text { T-DS } 80 \% / 80 \% \\
\text { RT delays } \geq 2 \text { days } 22 \% / 45 \% \\
\text { pCR in T3 } 22 / 69(32 \%) \\
\text { versus T4 } 5 / 27(18 \%) \\
5 \text { year: OS } 61 \% / 75 \%, \text { LRF } \\
16 \% / 17 \%, \text { DF } 16 \% / 21 \%\end{array}$ & $\begin{array}{l}\text { Grade } 3-4 \text { : overall } 42 \% / \\
51 \% \text {, } \\
\text { GI } 28 \% / 37 \% \text {, } \\
\text { blood/marrow } 9 \% / 12 \% \text {, } \\
\text { skin } 11 \% / 4 \% \text {, pain } 4 \% / 6 \% \\
\text { GU } 0 \% / 2 \% \text {, CVS } 2 \% / 0 \% \\
\text { Late toxicity } 6 \% \text { overall, } \\
\text { maximum severity grade } \\
34 \% / 6 \% \text {, grade } 40 \% / 2 \% \\
\text { mainly GI }\end{array}$ & $\begin{array}{l}93 \% \text { resected } \\
1 \text { death in each } \\
\text { arm }\end{array}$ \\
\hline [12] & II & $\begin{array}{l}\text { T3-4 resectable, } \\
\leq 15 \mathrm{~cm} \text { from anal } \\
\text { verge }\end{array}$ & $\begin{array}{l}\text { 5-FU } 2255 \text { days/week } \\
\text { Irino 50/week }\end{array}$ & $45 / 25$ & 74 & $13.7 \%$ & $\begin{array}{l}\text { Partial response } 61.6 \% \\
\text { Overall DS } 49.3 \% \\
\text { T-DS } 57.5 \% \\
\text { N-DS } 66.7 \% \\
\text { RT DR } 7 \% \\
\text { 5-FU DR } 10 \% \\
\text { Irino DR } 11 \%\end{array}$ & $\begin{array}{l}\text { Grade } 3 \text { : diarrhoea } 14 \% \text {, } \\
\text { asthenia } 9 \% \text {, abdominal } \\
\text { pain } 8 \% \text {, rectal mucositis } \\
8 \% \text {, anorexia } 5 \% \text {, } \\
\text { neutropenia } 4 \% \text {, anaemia } \\
3 \% \text {, constipation } 3 \% \\
\text { Grade } 4 \text { : neutropenia } 3 \% \text {, } \\
\text { TCP } 1 \%\end{array}$ & $\begin{array}{l}98.6 \% \text { resection } \\
58 \% \mathrm{TME} \\
59 \% \text { SSS } \\
84.9 \% \text { R0 } \\
1 \text { death (septic } \\
\text { shock, not } \\
\text { related to study } \\
\text { treatment) }\end{array}$ \\
\hline [13] & II & $\begin{array}{l}\mathrm{ct} / \mathrm{uT} 3 / 4 \text { or } \mathrm{N}+, \mathrm{M} 0 / \\
1, \\
\text { LR }\end{array}$ & $\begin{array}{l}\text { Cape } 500 \text { BD daily } \\
\text { Irino 50/week } \\
\text { ADJ: discretion }\end{array}$ & $50.4 / 28$ & $\begin{array}{l}36 \\
(\mathrm{M} 1 \\
n=3 \\
\text { LR } n= \\
3)\end{array}$ & $15 \%$ & $\begin{array}{l}\text { Microfoci remaining } 26 \% \\
\text { T-DS 55\% } \\
\text { N-DS } 63 \% \\
\text { RT DR } 14 \% \\
\text { Median received: } \\
\text { Cape } 100 \% \\
\text { Irino } 95 \% \\
\text { Actuarial } 2 \text { year OS } 83 \%\end{array}$ & $\begin{array}{l}\text { Grade 3: leukopenia } 19 \% \text {, } \\
\text { diarrhoea } 11 \% \text {, N\&V } 6 \% \text {, } \\
\text { fatigue } 3 \% \text {, raised ALT/ } \\
\text { AST } 3 \% \\
\text { Grade } 4 \text { : leukopenia } 6 \% \\
1 \text { VF arrest (reversed) }\end{array}$ & $\begin{array}{l}94 \% \text { resected, } \\
82 \% \text { SSS, } 100 \% \\
\text { R0 } \\
\text { DWH } 26 \%, \text { AL } \\
12 \% \\
2 \text { deaths } \\
\text { (sepsis) }\end{array}$ \\
\hline [14] & II & $\begin{array}{l}\text { mrT3 ( } \leq 2 \mathrm{~mm} \text { from } \\
\text { mesorectal fascia or } \\
\text { distal extent } \leq 5 \mathrm{~cm} \\
\text { from anal margin) or } \\
\text { mrT4, } \leq 15 \mathrm{~cm} \text { from } \\
\text { anal verge }\end{array}$ & $\begin{array}{l}\text { Cape } 650 \text { BD daily } \\
\text { Irino 60/week weeks } 1 \\
-4 \\
\text { ADJ: discretion }\end{array}$ & $45 / 25$ prone & 107 & $22 \%$ & $\begin{array}{l}\text { T-DS } 67 \% \\
\text { N-DS } 80 \% \\
\text { Mean dose delivered: RT } \\
97.4 \% \text {, Irino } 94.3 \% \text {, Cape } \\
87.9 \% \\
3 \text { years: LRF } 4 \% \text {, DFS } 63.5 \% \text {, } \\
\text { OS } 88.2 \%\end{array}$ & $\begin{array}{l}\text { Grade } 3 \text { : diarrhoea } 22 \% \text {, } \\
\text { fatigue } 11 \% \text {, radiation } \\
\text { dermatitis } 6 \% \text {, anorexia } \\
5 \% \text {, neutropenia } 5 \% \text {, } \\
\text { neutropenic fever/ } \\
\text { infection } 2 \%\end{array}$ & $\begin{array}{l}97 \% \text { resected at } \\
\text { median } \\
62 \text { days, 50\% } \\
\text { SSS, R1 } 8 \%\end{array}$ \\
\hline
\end{tabular}




\begin{tabular}{|c|c|c|c|c|c|c|c|c|c|}
\hline [15] & II & $\begin{array}{l}\mathrm{mr}+/-\mathrm{uT} 3 \text { or } \\
\text { resectable } \mathrm{T} 4\end{array}$ & $\begin{array}{l}\text { Cape } 825 \text { BD } 5 \text { days/ } \\
\text { week } \\
\text { Irino } 40 / \text { week } \\
\text { ADJ: Cape } 1250 \text { BD days } \\
1-14 / 3 \text { weeks } \times 6\end{array}$ & $50.4 / 28$ & 48 & $25 \%$ & $\begin{array}{l}\text { Dworak TRG } 318.2 \% \\
\text { T-DS 68.2\% } \\
\text { Relative dose intensity: } \\
\text { Cape } 93.4 \% \text {, Irino } 94.4 \% \\
\text { RT 100\% full dose, } 1 \\
\text { interruption } \\
5 \text { years: DFS 75\%, OS 93.6\%, } \\
\text { LRF 2\% (also had DF), DF } \\
\text { 25\% }\end{array}$ & $\begin{array}{l}\text { Grade 3: neutropenia, } \\
\text { infection, infection, } \\
\text { raised ALT, diarrhoea } \\
2.1 \% \\
\end{array}$ & $\begin{array}{l}94 \% \text { resected } \\
\text { (6\% cCR } \\
\text { declined, 9.1\% } \\
\text { transanal } \\
\text { excision), } 86 \% \\
\text { SSS } \\
2 \% \mathrm{AL}, 2 \% \mathrm{DWH}\end{array}$ \\
\hline [16] & II & cT3/4 N0-2 & $\begin{array}{l}\text { S1 } 40 \text { BD } 5 \text { days/week, } \\
\text { Irino } 80 \text { days } 1,8,22,29 \\
\text { ADJ: } \mathrm{S}-1 \text { + Irino (if } \\
\text { internal iliac or } \\
\text { obturator ypN+, } 9 \% \text { ) }\end{array}$ & $\begin{array}{l}45 / 25 \\
\text { prone (only perirectal } \\
\text { nodes included) }\end{array}$ & 67 & $34.7 \%$ & $\begin{array}{l}\text { 86.6\% completed CRT } \\
\text { (CALGB criteria) } \\
\text { G2 (good response) } 32.8 \% \\
\text { T-DS } 73.1 \% \\
\text { N-DS } 43.2 \%\end{array}$ & $\begin{array}{l}\text { Grade 3: diarrhoea, } \\
\text { leukopenia, neutropenia } \\
4.5 \% \text {, anorexia \& nausea } \\
1.5 \% \text {, TCP } 1.5 \%\end{array}$ & $\begin{array}{l}74.6 \% \text { SSS (all } \\
\text { had diverting } \\
\text { ileostomy } \\
\text { usually } \\
\text { reversed } \\
\text { at } 6-12 \\
\text { months due to } \\
\text { risk } \\
\text { of } A L)\end{array}$ \\
\hline [17] & II & cT3-4 or $\mathrm{N}+$ & $\begin{array}{l}\text { A: } 5 \text {-FU } 400+\text { LV } 20 \\
\text { bolus days } 1-3 / 28 \text { days } \\
\times 2 \\
\text { B: S1 } 35 \text { BD } 5 \text { days/ } \\
\text { week + Irino } 40 / \text { week } \\
\times 5 \\
\text { ADJ: } 5 \text {-FU }\end{array}$ & $\begin{array}{l}45-50.4 / \\
25-28\end{array}$ & 142 & $\begin{array}{l}\text { A: } 17.2 \% \\
\text { B: } 24.2 \% \\
(P=0.1)\end{array}$ & & $\begin{array}{l}\text { Grade } 3 / 4 \\
\text { A: } 1.4 \% \\
\text { B: } 7 \%(P=0.095)\end{array}$ & 91.5\% TME \\
\hline [18] & II & $\begin{array}{l}\text { Locally recurrent, } \\
\text { unirradiated M0/1 }\end{array}$ & $\begin{array}{l}\text { Cape } 625 \text { BD } 5 \text { days/ } \\
\text { week }+ \text { Irino 50/week } \\
\text { weeks } 1-5\end{array}$ & $\begin{array}{l}45 / 25 \\
\text { Boost } 10-16 / 5-8\end{array}$ & 71 & $\begin{array}{l}50 \% \\
(7 / 14)\end{array}$ & $\begin{array}{l}\text { cCR } 5.6 \% \\
\text { Complete symptom relief } \\
56.6 \% \text { of } 53 \text { symptomatic } \\
\text { patients, partial in } 32.1 \%\end{array}$ & \multicolumn{2}{|c|}{$\begin{array}{l}\text { Grade 3: diarrhoea } 22.5 \%, \mathrm{R} 0 \text { resection } \\
\text { dermatitis } 9.9 \%, \quad 20 \% \\
\text { leukopenia } 5.6 \% \text {, cystitis } \\
\text { 4.2\%, } \\
\text { Grade 4: leukopenia } 2.8 \%\end{array}$} \\
\hline
\end{tabular}


Table 2

Phase III randomised controlled trials of neoadjuvant chemoradiotherapy (CRT) with fluoropyrimidine + oxaliplatin

\begin{tabular}{|c|c|c|c|c|c|c|c|c|}
\hline $\begin{array}{l}\text { Reference } \\
\text { Trial }\end{array}$ & Disease stage* & $\begin{array}{l}\text { Systemic treatment } \\
\left(\mathrm{mg} / \mathrm{m}^{2}\right)\end{array}$ & $\begin{array}{l}\mathrm{RT}(\mathrm{Gy} / \\
\text { fractions)† }\end{array}$ & $n$ & $\mathrm{pCR}$ & Other end points & $\begin{array}{l}\text { Grade } 3-4 \text { toxicity } \\
\text { (non-surgical) }\end{array}$ & Surgical outcomes \\
\hline $\begin{array}{l}28] \\
\text { ACCORD } \\
12 / \\
0405- \\
\text { Prodige } \\
2\end{array}$ & $\begin{array}{l}\text { T2 (ant/lower) } \\
\text { T3 } \\
\text { T4 (resectable) } \\
\text { Accessible to DRE }\end{array}$ & $\begin{array}{l}\text { A: Cape } 800 \text { BD } 5 \text { days/week } \\
\text { B: + Oxali } 50 / \text { week } \\
\text { ADJ: discretion }\end{array}$ & $\begin{array}{l}A: 45 / 25 \\
B: 50 / 25\end{array}$ & $\begin{array}{l}\text { A: } 293 \\
\text { B: } 291\end{array}$ & $\begin{array}{l}3 \text { A: } 13.9 \% \\
1 \text { B: } 19.2 \% \\
(P=0.09)\end{array}$ & $\begin{array}{l}\text { Full dose RT: } 100 \% / \\
87 \% \\
\text { Dworak Score } 3 \text { or } 2 \text { : } \\
28.9 \% / 39.4 \% \\
(P=0.008) \\
3 \text { years: NSD in } \\
\text { LRF } 6.1 \% / 4.4 \% \text {, OS } \\
87.6 \% / 88.3 \% \text { DFS } \\
67.9 \% / 72.7 \%[29]\end{array}$ & $\begin{array}{l}\text { Overall } 10.9 \% / 25.4 \% \\
(P<0.001), \text { diarrhoea } \\
3.2 \% / 12.6 \%(P<0.001) . \\
\text { Grade 3: fatigue } 0.8 \% / \\
5.1 \%(P=0.004)\end{array}$ & $\begin{array}{l}97 \% \text { TME } \\
\text { CRM } \leq 2 \mathrm{~mm} 19.3 \% / 9.9 \% \\
(P=0.022) \\
\text { NSD in rates of surgery (98/ } \\
98.6 \%), \text { conservative surgery } \\
\text { rate (75\% overall), } \\
\text { Abdominopelvic distant } \\
\text { metastases at surgery }(4.2 \% / \\
2.8 \%, P=0.4) \text { or } \mathrm{R} 1(12.7 \% / \\
7.7 \%, P=0.17) .\end{array}$ \\
\hline $\begin{array}{l}{[30]} \\
\text { STAR-01 }\end{array}$ & $\begin{array}{l}\text { cT3-4 } \\
\text { \&/or N1-2 } \\
\text { Resectable, within } \\
12 \mathrm{~cm} \text { anal verge }\end{array}$ & $\begin{array}{l}\text { A: 5-FU 225/day } \\
\text { B: + Oxali 60/week } \\
\text { ADJ: 5-FU based }\end{array}$ & $50.4 / 28$ & $\begin{array}{l}\text { A: } 379 \\
\text { B: } 368\end{array}$ & $\begin{array}{l}9 \text { A: } 16 \% \\
8 \text { B: } 16 \% \\
(P=0.9)\end{array}$ & $\begin{array}{l}\text { Full dose 5-FU: } 90 \% / \\
80 \%(P<0.001) \\
\text { Full dose RT: } 92 \% / 84 \% \\
(P<0.001) \\
\text {-discontinued due to } \\
\text { toxicity } 4 \% / 17 \% \\
\text { Dworak TRG 3: 39\%/ } \\
\text { 45\% }\end{array}$ & $\begin{array}{l}\text { Overall } 8 \% / 24 \% \\
(P<0.001) \text {, diarrhoea } 4 \% / \\
15 \%(P<0.001) \text {; nausea, } \\
\text { abdominal pain } 0 \% / 2 \% \\
(P=0.012), \text { radiation } \\
\text { dermatitis } 2 \% / 5 \% \\
(P=0.037), \text { SN } 0 \% / 1 \% \\
(P=0.026), \text { asthenia } 0 \% / \\
3 \%(P<0.001) \\
3 \text { deaths }(1 / 2)\end{array}$ & $\begin{array}{l}\text { Intra-abdominal distant } \\
\text { metastases at surgery } 2.9 \% \text { | } \\
0.5 \%(P=0.014) \\
\text { NSD in rates of surgery ( } 96 \% / \\
95 \%) \text {, APR or Hartmann's } \\
(21 \% / 19 \%), \text { CRM } \leq 1 \mathrm{~mm} \text { ( } 7 \% \text { | } \\
4 \%), \text { R0 } 94 \% / 97 \% \text {, } \\
\text { postoperative complications } \\
(22 \% / 24 \%), 60 \text { day mortality } \\
(1 \% / 1 \%) .\end{array}$ \\
\hline $\begin{array}{l}\text { CA1] } \\
\text { ARO/ } \\
\text { AIO-04 }\end{array}$ & $\begin{array}{l}\text { cT3-4 or } \mathrm{cN}+\text {, within } \\
12 \mathrm{~cm} \text { anal verge }\end{array}$ & $\begin{array}{l}\text { A: } 5 \text {-FU } 1000 \text { days } 1-5,29 \\
-33 \\
\text { B: } 5 \text {-FU } 250 \text { days } 1-14,22 \\
-35+\text { Oxali } 50 \text { days } \\
1,8,22,29 \\
\text { ADJ: } \\
\text { A: 5-FU } \\
\text { B: } 5-F U+\text { Oxali }\end{array}$ & $50.4 / 28$ & $\begin{array}{l}\text { A: } 623 \\
\text { B: } 613\end{array}$ & $\begin{array}{l}3 \text { A: } 13 \% \\
3 \text { B: } 17 \% \\
(P=0.038)\end{array}$ & $\begin{array}{l}\text { Full dose: RT } 96 \% / 94 \% \text {, } \\
\text { NA chemo } 79 \% / 85 \% \\
\text { ADJ chemo completed } \\
65 \% / 44 \% \text {. } \\
3 \text { years: DFS } 71.2 \% / \\
75.9 \%(P=0.03), \text { NSD } \\
\text { in OS } 88 \% / 88.7 \% \text { or } \\
\text { LRF } 4.6 \% / 2.9 \%[32]\end{array}$ & $\begin{array}{l}\text { Overall } 20 \% / 23 \% \text {, } \\
\text { haematological } 6 \% / 5 \% \text {, GI } \\
\text { overall } 15 \% / 20 \% \text {, } \\
\text { diarrhoea } 8 \% / 12 \% \\
6 \text { deaths }(2 / 4)\end{array}$ & $\begin{array}{l}\text { R0 } 95 \% / 94 \%, \text { CRM } \leq 1 \mathrm{~mm} \\
6 \% / 5 \% \text {, APR } 24 \% / 25 \% \text {, good } \\
\text { quality TME } 77 \% / 76 \% \\
60 \text { day mortality } 4 / 3 \text { deaths } \\
\text { Complications grade } 3-4 \\
10 \% / 13 \% \text {, AL } 5 \% / 7 \%\end{array}$ \\
\hline $\begin{array}{l}\text { [33] } \\
\text { PETACC- } \\
6\end{array}$ & $\begin{array}{l}\mathrm{T} 3 / 4 \text { \&/or } \mathrm{N}+ \\
\text { Resectable/potentially } \\
\text { resectable, within } \\
12 \mathrm{~cm} \text { of anal verge }\end{array}$ & $\begin{array}{l}\text { A: Cape } 825 \text { BD daily } \\
\text { B: + Oxali 50/week } \\
\text { A: Cape } \\
\text { B: Cape + Oxali }\end{array}$ & $\begin{array}{l}45 / 25 \\
+ \text { optional } \\
\text { boost to } 50.4\end{array}$ & $\begin{array}{l}\text { A: } 547 \\
\text { B: } 547\end{array}$ & $\begin{array}{l}7 \text { A: } 11.3 \% \\
7 \text { B: } 13.3 \% \\
(P=0.31)\end{array}$ & $\begin{array}{l}\geq 45 \text { Gy } 98 \% / 92 \% \\
>90 \% \text { chemo } 91 \% / 63 \% \\
\text { DS } 43.5 \% / 41.5 \% \\
3 \text { year DFS } 74.5 \% / \\
73.9 \% \\
\text { LRF + DF } 20 \% / 18 \%\end{array}$ & $\begin{array}{l}\text { Toxicity (undefined) } \\
7.7 \% / 16.5 \% \\
\text { Deaths without } \\
\text { progression } 15 \text { versus } 26 \\
\text { [34] }\end{array}$ & $\begin{array}{l}\text { R0 92\%/86.3\% } \\
\text { SSS 70\%/65\% }(P=0.09)\end{array}$ \\
\hline $\begin{array}{l}\text { [35] } \\
\text { NSAPB } \\
\text { R-04 }\end{array}$ & $\begin{array}{l}\text { CT3-4,N0 or } \\
\text { cT1-4,N1-2 } \\
\text { Within } 12 \mathrm{~cm} \text { anal } \\
\text { verge, palpable on DRE } \\
\text { or accessible by procto- } \\
\text { or sigmoidoscope }\end{array}$ & $\begin{array}{l}\text { A: 5-FU 225/day } \\
\text { B: Cape } 825 \text { BD } \\
\text { Post protocol amendment } \\
\text { C: } 5 \text {-FU } 2255 \text { days/week } \\
\text { D: C + Oxali } 50 \\
\text { E: Cape } 825 \text { BD } 5 \text { days/week } \\
\text { F: E + Oxali 50/week } \\
\text { ADJ: not defined }\end{array}$ & $\begin{array}{l}45 / 25 \\
\text { Boost: T3 } \\
\text { non-fixed, } \\
\text { non-distal } \\
5.4 / 3 \\
\text { T4 fixed } \\
+/ \text { or distal } \\
10.8 / 3\end{array}$ & $\begin{array}{l}\text { A: } 147 \\
\text { B: } 146 \\
\text { C: } 330 \\
\text { D: } \\
329 \\
\text { E: } 326 \\
\text { F: } 330\end{array}$ & $\begin{array}{l}7 \text { No Oxali } \\
617.8 \% \\
0 \text { With Oxali } \\
19.5 \% \\
(P=0.42) \\
6 \\
0\end{array}$ & $\begin{array}{l}\text { Dose delivered } \\
\text { fluoropyrimidine } \\
\text { C/E }>\mathrm{D} / \mathrm{F}(P<0.05) \\
\text { No Oxali versus with } \\
\text { Oxali: DS } 23.5 \% / 17.9 \% \\
(P=0.2) . \\
3 \text { year LRF } 12.1 \% / \\
11.2 \% \\
5 \text { year: DFS } 64.2 \% /\end{array}$ & $\begin{array}{l}\text { No Oxali versus with } \\
\text { Oxali: } \\
\text { diarrhoea grade } 3-5 \\
6.9 \% / 16.5 \%(P<0.001) \text {, } \\
2 \text { in CAPOX arm died } \\
\text { Arms C/D/E/F: } \\
\text { Grade } 3(\%) \text { : } \\
\text { nausea } 0.3 / 0.6 / 1.3 / 2.2 \text {, } \\
\text { fatigue } 1.3 / 4 / 2.2 / 5.9 \text {, }\end{array}$ & $\begin{array}{l}\text { No Oxali versus with Oxali: } \\
\text { SSS } 61 \% / 57.8 \%(P=0.24) \\
\text { NSD in postoperative } \\
\text { complications arms A-F }(\%) \text { : } \\
\text { Overall } 30.7 / 37.4 / 37.3 / 37.5 / \\
36.2 / 40.5 \\
\text { AL } 2.1 / 3.1 / 1.3 / 3.6 / 3.0 / 1.6 \\
\text { Death } 0.7 / 0.0 / 0.3 / 0.7 / 0.7 / 0.0\end{array}$ \\
\hline
\end{tabular}




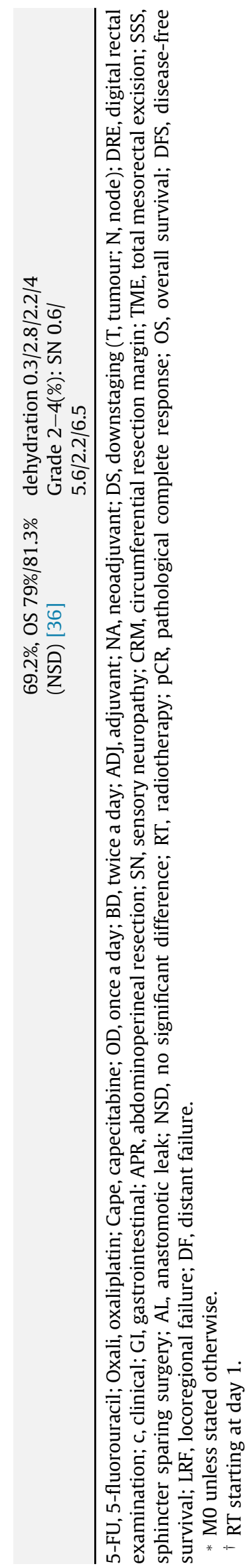

analysis, the CAO/ARO/AIO-04 trial exploratory subset analysis of DFS indicated significant benefit in the investigational group for $\mathrm{cNO}$ disease (hazard ratio 0.56; $95 \%$ confidence interval $0.36-0.86$ ), but no difference was seen with pathological ypTNM subgroups (hazard ratio 1.20; 95\% confidence interval 0.43-3.36). CAO/ARO/AIO-04 showed no significant difference in 3 year overall survival (88\%, 95\% confidence interval $85.3-90.7 \%$ versus $88.7 \%, 95 \%$ confidence interval $86-91.3 \%$ ) or 3 year LRF $(4.6 \%, 95 \%$ confidence interval $2.9-6.4 \%$ versus $2.9 \%$, $95 \%$ confidence interval 1.5-4.3\%) after $\mathrm{R} 0 / 1$ resection in control and investigational groups, respectively. Comparable results were seen in the ACCORD trial with 3 year overall survival $87.6 \%$ versus $88.3 \%$ (hazard ratio 0.94 ; $95 \%$ confidence interval $0.59-1.48$ ). Three year LRF was $6.1 \%$ versus $4.4 \%$, whereas Allegra et al. [36] in the NSABP R-04 trial found higher rates of $12.1 \%$ versus $11.2 \%$ without and with oxaliplatin, respectively.

Surgical outcomes were not significantly different with the addition of oxaliplatin. In ACCORD, exploratory analysis revealed a difference in CRM $\leq 2 \mathrm{~mm}$ of $19.3 \%$ versus $9.9 \%$ $(P=0.022)$ without and with oxaliplatin, but this difference was not seen at CRM $\leq 1 \mathrm{~mm}[28,30,31]$. Whereas surgical toxicity was not altered with the addition of oxaliplatin, ACCORD, STAR-01 and NSABP R-04 all reported significantly increased CRT-related toxicity. Grade 3-4 diarrhoea was particularly problematic with rates up to $25.4 \%$ versus $10.9 \%$ without oxaliplatin in ACCORD and two deaths attributable in NSABP. Four trials showed a higher proportion of patients not receiving full dose chemotherapy or radiotherapy in the experimental arm $[28,30,34,35]$. The ACCORD trial assessed late toxicity at 3 years and found no difference between the arms in terms of bowel continence, erectile dysfunction or social life disturbance [38].

One randomised phase II trial [39] has compared the pCR rates of CRT with CAPIRI (capecitabine $600 \mathrm{mg} / \mathrm{m}^{2}$ BD 5 days/week and irinotecan $50 \mathrm{mg} / \mathrm{m}^{2}$ weekly four doses) or CAPOX (capecitabine $825 \mathrm{mg} / \mathrm{m}^{2}$ BD 5 days/week and oxaliplatin $50 \mathrm{mg} / \mathrm{m}^{2}$ weekly five doses). The pCR rate in the latter group was higher at $23.1 \%$ (95\% confidence interval $12.5-36.8 \%$ ) versus $11.8 \%$ (95\% confidence interval 4.4-23.9\%), with toxicity levels comparable and tolerable in both groups. Follow-up to 4 years revealed that both regimens showed efficacy with LRF 16\% versus $18 \%$, DFS $68 \%$ versus $62 \%$ and overall survival $85 \%$ (95\% confidence interval $66-94 \%$ ) versus $75 \%$ (95\% confidence interval $60-85 \%$ ), respectively [126]. The contradictory observation of higher overall survival in the irinotecan arm was noted, but this study was not designed to permit direct statistical comparisons between the two arms.

In conclusion, the addition of oxaliplatin to standard neoadjuvant CRT has shown improved PCR rates in one meta-analysis of four trials. Although this has not translated into improvement in survival measures to date, publication of long-term data is still awaited for two large phase III trials. The regimen does seem to be associated with increased acute toxicity and reduced treatment compliance. Nevertheless, several trials have added biological agents to oxaliplatin, fluoropyrimidine and radiotherapy. Pre-clinical 
Table 3

Clinical trials of bevacizumab (BEV) with neoadjuvant chemoradiotherapy (CRT) for rectal cancer

\begin{tabular}{|c|c|c|c|c|c|c|c|c|c|}
\hline \multicolumn{2}{|c|}{ Reference Stage* } & $\begin{array}{l}\text { Systemic } \\
\text { treatment }\end{array}$ & $\begin{array}{l}\mathrm{RT}(\mathrm{Gy} / \\
\text { fractions }) \dagger\end{array}$ & Surgery & $n$ & $\mathrm{pCR}$ & Other end points & Grade $3-4$ toxicity (non-surgical) & Surgical outcomes \\
\hline \multicolumn{10}{|c|}{ Bevacizumab with fluoropyrimidine-based neoadjuvant CRT (phase II) } \\
\hline [45] & cT3/4 & $\begin{array}{l}\text { Cape } 825 \text { BD } \\
\text { daily } \\
\text { BEV } 5 \text {, days } \\
-14,1,15,29\end{array}$ & $50 / 25$ & $\begin{array}{l}6-10 \\
\text { weeks }\end{array}$ & 23 & $\begin{array}{l}9.5 \% \\
(2 / 21)\end{array}$ & $\begin{array}{l}\text { T-DS: } 85.7 \%(18 / \\
\text { 21) }\end{array}$ & $\begin{array}{l}\text { Grade } 3 \text { : skin } 17 \% \text {, diarrhoea } 9 \% \text {, } \\
\text { tenesmus } 4 \% \\
\text { Grade } 4: \text { anal mucositis } 4 \% \\
9 \% \text { small bowel, } 4 \% \text { rectal wall } \\
\text { perforation } \\
1 \text { death secondary to enteritis with } \\
\text { uncontrolled bleeding }\end{array}$ & $\begin{array}{l}\text { 96\% resected, } 45 \% \text { SSS, } 91 \% \text { R0 } \\
\text { Rectovaginal fistula, peri- } \\
\text { operative bleeding, pulmonary } \\
\text { embolism, perineal dehiscence } \\
5 \%\end{array}$ \\
\hline [46] & $\mathrm{cT} 3 / 4$ & $\begin{array}{l}\text { 5-FU 225/day } \\
\text { BEV 5, days } \\
-14,1,15,29 \\
\text { DL1:5 (PII dose) } \\
\text { DL2:10 }\end{array}$ & $50.4 / 28$ & $\begin{array}{l}7-10 \\
\text { weeks }\end{array}$ & $\begin{array}{l}32 \\
\text { PI: } 11 \\
\text { PII: } 21\end{array}$ & $\begin{array}{l}15.6 \% \\
(5 / 32)\end{array}$ & $\begin{array}{l}\text { T-DS } 50 \% \\
\text { N-DS } 56.5 \% \\
\text { Actuarial } 5 \text { year } \\
\text { OS 95\%, DFS } \\
68.9 \%[47]\end{array}$ & $\begin{array}{l}\text { Grade } 3 \text { : overall GI } 41 \% \text {, diarrhoea } 22 \% \text {, } \\
\text { hypertension } 9 \% \text {, Skin/RT dermatitis } 6 \% \text {; } \\
\text { PPE, neurologic, wound separation } 3 \%\end{array}$ & $\begin{array}{l}\text { 100\% resected, } 94 \% \text { R0 } \\
\text { Wound infection } 9 \% \text {; } \\
\text { haematoma, DWH 6\%; AL, PE 3\% }\end{array}$ \\
\hline [48] & $\mathrm{T} 3 / 4$ or $\mathrm{N} 1$ & $\begin{array}{l}\text { Cape } 900 \text { BD } 5 \\
\text { days/week } \\
\text { BEV 5, days } \\
1,15,29 \\
\text { ADJ: discretion }\end{array}$ & $50.4 / 28$ & $\begin{array}{l}6-10 \\
\text { weeks } \\
\text { TME }\end{array}$ & 25 & $\begin{array}{l}32.0 \% \\
(8 / 25)\end{array}$ & $\begin{array}{l}\text { Microscopic } \\
\text { residual 24\% } \\
\text { Overall DS 82\% } \\
\text { T-DS 64\%, N-DS } \\
79 \% \\
\text { Median follow- } \\
\text { up } 22.7 \text { months: } \\
\text { 4\% LRF, 12\% DF } \\
\text { Actuarial 2 year } \\
\text { DFS 77.3\% }\end{array}$ & Grade 3: perianal desquamation $4 \%$ & $\begin{array}{l}100 \% \text { resection, } 100 \% \text { R0, } 72 \% \text { SSS } \\
\text { DWH, perineal wound } \\
\text { dehiscence (required further } \\
\text { surgery), AL } 8 \% \text {; anastomotic } \\
\text { dehiscence (required further } \\
\text { surgery), superficial wound } \\
\text { abscess } 4 \%\end{array}$ \\
\hline [49] & $\begin{array}{l}\mathrm{T} 3 \text { or operable } \\
\mathrm{T} 4, \mathrm{NO} /+\leq 12 \mathrm{~cm} \\
\text { anal margin }\end{array}$ & $\begin{array}{l}\text { Induction: } 4 \times \\
21 \text { day cycles } \\
\text { Cape } 1000 \text { BD } \\
\text { days } 1-15 \\
\text { OX } 130 \text { day } 1 \\
\text { BEV } 7.5 \\
\text { CRT: } \\
\text { Cape } 825 \text { BD } \\
\text { daily } \\
\text { BEV 5, days } \\
1,15,29 \\
\text { ADJ: CAPOX x4 } \\
\text { recommended }\end{array}$ & $50.4 / 28$ & $\begin{array}{l}6-8 \\
\text { weeks } \\
\text { TME }\end{array}$ & 47 & $\begin{array}{l}35.6 \% \\
(16 / 45)\end{array}$ & $\begin{array}{l}\text { Dworak TRG } \\
37.8 \% \\
87 \% \text { completed } \\
\text { NA treatment }\end{array}$ & $\begin{array}{l}\text { CRT phase: } \\
\text { Grade 3: lymphopenia, } \\
\text { hypertriglyceridemia, tenesmus 3\% }\end{array}$ & $\begin{array}{l}96 \% \text { resected, } 97.8 \% \text { R0, } 67 \% \text { SSS } \\
58 \% \text { postoperative } \\
\text { complications: } \\
\text { wound infection } 22 \% \text {, intra- } \\
\text { abdominal infection } 16 \% \text {, AL } 11 \% \text {, } \\
\text { stoma complications } 4 \% \text {, } \\
\text { other } 22 \% \\
24 \% \text { further surgery }\end{array}$ \\
\hline [50] & II/III & $\begin{array}{l}\text { Non- } \\
\text { randomised } \\
\text { Cohort A: } \\
\text { NACRT 5-FU } \\
\text { 225/day, BEV 5, } \\
\text { days 1,15 } \\
\text { Cohort B: }\end{array}$ & $50.4 / 28$ & $\begin{array}{l}\text { A: } 2-8 \\
\text { weeks }\end{array}$ & $\begin{array}{l}66 \\
\text { A: } 35 \\
\text { B: } 31\end{array}$ & $\begin{array}{l}\text { A: } 29 \% \\
(10 / 35)\end{array}$ & $\begin{array}{l}\text { A: Microscopic } \\
\text { residual } 11 \% \\
\text { Discontinued } \\
\text { treatment due to } \\
\text { toxicity: } \\
\text { A } 23 \% \text {, B } 16 \% \\
\text { Started ADJ }\end{array}$ & $\begin{array}{l}\text { A (neoadjuvant CRT phase) } \\
\text { Grade 3: diarrhoea } 6 \% \text {; neutropenia, } \\
\text { fatigue, dehydration } 3 \% \text {, } \\
\text { Grade } 4 \text { : TCP } 9 \% \\
\text { B (ADJ CRT phase) } \\
\text { Grade 3: diarrhoea } 26 \% \text {, rash/ }\end{array}$ & $\begin{array}{l}\text { A: } 89 \% \text { resected, } 32 \% \text { TME, 39\% } \\
\text { SSS } \\
\text { Complications: } \\
\text { A: grade } 4 \text { rectal bleed, } \\
\text { perforation, pelvic infection 3\%. } \\
\text { B: perforation, perianal infection }\end{array}$ \\
\hline
\end{tabular}




\begin{tabular}{|c|c|c|c|c|c|c|c|c|c|}
\hline & & $\begin{array}{l}\text { ADJ CRT 5-FU } \\
225 / \text { day, BEV } 5 \\
2-\text { weekly from } \\
\text { day } 1 . \\
\text { ADJ: modified } \\
\text { FOLFOX6 } \\
\text { x4 + BEV for } 1 \\
\text { year }\end{array}$ & & & & & $\begin{array}{l}\text { chemo: A 57\%, B } \\
84 \%\end{array}$ & $\begin{array}{l}\text { desquamation } 13 \% \text {; dehydration, } \\
\text { mucositis } 6 \% \text {, fatigue } 3 \%\end{array}$ & $\begin{array}{l}6 \% \text {; anal wound dehiscence, } \\
\text { rectovaginal fistula } 3 \%\end{array}$ \\
\hline [51] & $\begin{array}{l}\text { II/III likely } \\
\text { resectable }\end{array}$ & $\begin{array}{l}\text { Cape } 825 \text { BD } \\
\text { daily } \\
\text { BEV 5, days } \\
-14,1,15,29 \\
\text { ADJ: Cape x4 } \\
\text { (R0) or x6 (R1) }\end{array}$ & $50.4 / 28$ & $\begin{array}{l}6-8 \\
\text { weeks } \\
\text { TME }\end{array}$ & 61 & $\begin{array}{l}13.3 \% \\
(8 / 60)\end{array}$ & $\begin{array}{l}\text { Dworak TRG3 } \\
15.0 \% \\
\text { Overall DS 75\% } \\
\text { T-DS } 46.7 \% \\
\text { N-DS } 65 \% \\
\text { Dose intensity: } \\
\text { RT } 100 \% \text {, BEV } \\
95.1 \%, \text { Cape } \\
\text { ( } \geq 95 \% \text { ) } 91 \% \\
3 \text { year OS } 80.9 \% \text {, } \\
\text { DFS } 74.1 \%, \text { LRF } \\
5.6 \%[52]\end{array}$ & $\begin{array}{l}\text { Grade } 3 \text { : dermatitis } 9.8 \% \text {, proteinuria } \\
6.5 \% \text {, leukopenia } 4.9 \% \text {; diarrhoea, } \\
\text { vascular, hypertension } 1.6 \% \\
\text { Grade } 4 \text { : vascular } 1.6 \%\end{array}$ & $\begin{array}{l}95 \% \text { radical resection, } 70 \% \text { SSS } \\
62.3 \% \text { peri-operative } \\
\text { complications: } \\
\text { DWH } 30 \% \text {, infection/abscess } 20 \% \text {, } \\
\text { AL } 11.7 \% \text {, pneumothorax } 1.7 \% \\
10 \% \text { further surgery }\end{array}$ \\
\hline [53] & $\begin{array}{l}\text { T3Nx potentially } \\
\text { resectable }\end{array}$ & $\begin{array}{l}\text { Cape } 825 \text { BD } 5 \\
\text { days/week } \\
\text { weeks } 1-4 \\
\text { BEV } 5 \text {, days } \\
1,15,29\end{array}$ & $45 / 25$ & $\begin{array}{l}6-8 \\
\text { weeks } \\
\text { TME }\end{array}$ & 8 & $\begin{array}{l}25 \% \\
(2 / 8)\end{array}$ & $\begin{array}{l}\text { T-DS 37.5\% } \\
\text { Full dose: RT } \\
87.5 \% \text {, Cape } \\
87.5 \% \text {, BEV } 75 \%\end{array}$ & $\begin{array}{l}\text { Grade } 3 / 4 \text { : perianal bleeding, perianal } \\
\text { or abdominal pain, diarrhoea } 25 \% \text {, } \\
\text { anaemia } 12.5 \% \\
\text { Trial terminated as } 50 \% \text { had grade } 3 / 4 \\
\text { toxicity }\end{array}$ & $\begin{array}{l}88 \% \text { resected } \\
\text { Ileus grade } 3 \text {, perineal wound } \\
\text { dehiscence } 14 \%\end{array}$ \\
\hline [54] & $\mathrm{T} 2 \mathrm{~N}+, \mathrm{T} 3 / 4$ & $\begin{array}{l}\text { Cape } 825 \text { BD } \\
\text { daily, } \\
\text { BEV 5, days } \\
-14,1,15,29 \\
\text { ADJ: 5-FU/LV } \\
\text { x12 } \pm \text { BEV }\end{array}$ & $50.4 / 28$ & $\begin{array}{l}6-8 \\
\text { weeks } \\
\text { TME }\end{array}$ & 43 & $\begin{array}{l}14.0 \% \\
(6 / 43)\end{array}$ & $\begin{array}{l}\text { Microscopic } \\
\text { residual } 16.3 \% \\
\text { T-DS } 34.9 \% \\
\text { N-DS } 41.9 \% \\
\text { Discontinued } \\
\text { CRT } 9.5 \% \\
\text { Median follow- } \\
\text { up } 16.7 \text { months: } \\
\text { LRF } 11.6 \% \text {, DF } 7 \% \\
\text { Actuarial } 3 \text { year } \\
\text { DFS } 75.4 \%\end{array}$ & $\begin{array}{l}\text { Grade 3: diarrhoea } 7.1 \% \text {, neutropenia } \\
\text { 4.7\%; asthenia, hypokalaemia } 2.4 \%\end{array}$ & $\begin{array}{l}93 \% \text { resected, } 95 \% \text { R0, } 78 \% \text { SSS } \\
\text { Bowel perforation ( } 81 \text { days after } \\
\text { BEV, died), anastomotic failure, } \\
\text { abscess } 2.5 \%\end{array}$ \\
\hline [55] & $\begin{array}{l}\text { II/III } \\
\text { MRI } \\
<15 \mathrm{~cm} \text { anal } \\
\text { verge }\end{array}$ & $\begin{array}{l}\text { Randomised } \\
1: 1 \\
\text { A: Cape } 825 \text { BD } \\
\text { daily, BEV 5, } \\
\text { days } 1,15,29 \\
\text { B: Cape } 825 \text { BD } \\
\text { daily }\end{array}$ & $45 / 25$ & $\begin{array}{l}6-8 \\
\text { weeks } \\
\text { TME or } \\
\text { PME }\end{array}$ & $\begin{array}{l}90 \\
\text { A: } 44 \\
\text { B: } 46\end{array}$ & $\begin{array}{l}\text { A: } 16 \% \\
(7 / 44) \\
\text { B: } 11 \% \\
(5 / 46) \\
P=0.54\end{array}$ & $\begin{array}{l}\text { A/B } \\
\text { Mandard TRG } 1 \\
-2: 36 \% / 44 \% \\
\text { T-DS: } 59 \% / 39 \% \\
(P=0.04) \\
\text { CRT complete } \\
\text { 93\%/93\% } \\
\text { Median follow- } \\
\text { up } 18 \text { months: } \\
\text { LRF 0/2\%, DF } 14 \% / \\
\text { 13\%, Death due to } \\
\text { disease } 5 \% / 0 \%\end{array}$ & $\begin{array}{l}\text { Grade 3: overall } 16 \% / 13 \%(P=0.70) \text {, } \\
\text { asthenia/fatigue } 2 \% / 0 \% \text {, anorectal } \\
\text { discomfort } 2 \% / 0 \% \text {, PPE } 0 \% / 2 \%\end{array}$ & $\begin{array}{l}98 \% / 100 \% \text { resected, } 77 \% / 78 \% \\
\text { TME, } 61 \% / 67 \% \text { SSS } \\
\text { Overall postoperative } \\
\text { complications } 43 \% / 39 \% \text {, } \\
\text { anastomotic dehiscence } \\
\text { requiring surgery } 15.9 \% / 6.5 \% \\
\text { Intra-abdominal distant } \\
\text { metastases at surgery } 0 \% / 9 \%\end{array}$ \\
\hline
\end{tabular}




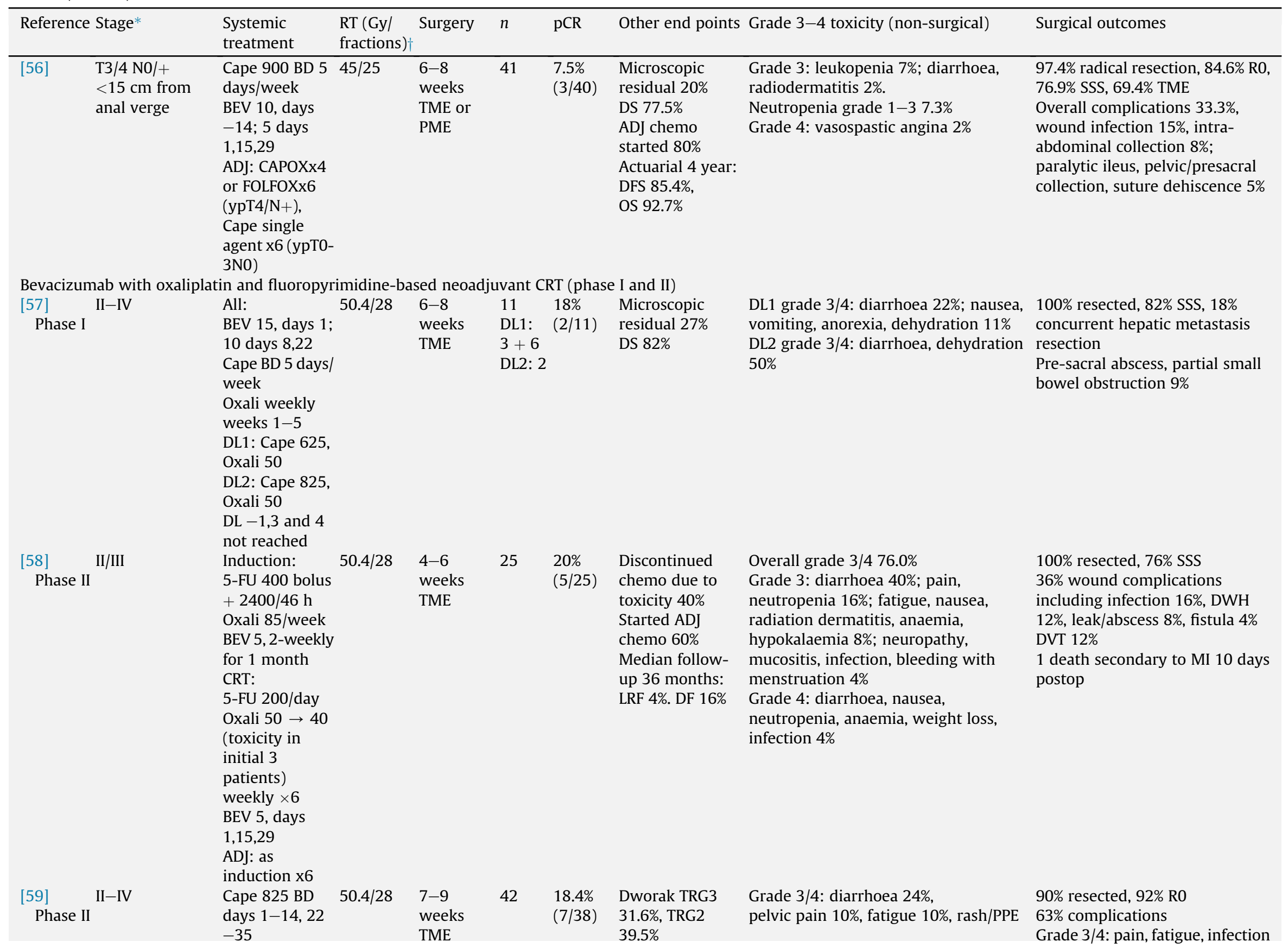


Oxali 50 days

$1,8,22,29$

BEV 5, days

$-14,1,15,29$

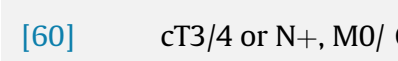

Phase II 1 (single

resectable liver -35

metastasis), Oxali 50 days

$<16 \mathrm{~cm}$ from $1,8,22,29$

anal verge BEV 5, days

$1,15,29$

ADJ:

Recommended

Cape 1250 BD

days $1-14 / 21$

$\mathrm{x} 4$

$\begin{array}{ccc}{[61]} & \mathrm{T} 3 / 4, \leq 12 \mathrm{~cm} & \text { Cape } 825 \\ \text { Phase II from anal verge } & \text { days/week }\end{array}$

Oxali 50

weekly

BEV 5, days

$1,15,29$

ADJ (if

macroscopic

complete

resection):

2 weekly 5 -FU/

LV/BEV 5

$\mathrm{x} 12+$ Oxali $\mathrm{x} 9$

Bevacizumab with fluoropyrimidine-based neoadjuvant CRT + other agents (phase I and II)

[63] T3/4 or $\mathrm{N}+$, All D :

AIl DL:

Phase I $\leq 12 \mathrm{~cm}$ ana

verge

$\times 3$

Cape BD 5 days

week DL1: 650

DL2-4: 825

Erlotinib (mg)

OD

DL1-2: 50

weeks 1-3

DL3: 50

weeks $1-6$

DL4: 100

weeks $1-6$

ADJ: Discretion

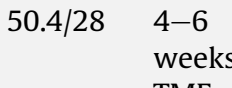

weeks

69

$90 \%$

6-8

weeks

TME

50.4/28 Minimum 18

$44 \%$

9 weeks DL1: $2(8 / 18)$

9 we

DL2: 4

DL3: 4

DL4: 8

DFS 94\%
Mean dose

intensity: BEV

$7 \%$, hypertension $5 \%$; pelvic infection,

95\%, Oxali $97 \%$,

Cape 91\%, RT 97\%

$17.4 \%$ DS $44.9 \%$

(12/69) Mean dose

intensity: BEV

$98.6 \%$, Oxali

97.8\%, RT $98.4 \%$.

Full dose Cape

T-DS 59.3\%

(9/54) N-DS 66\%

ADJ chemo

started $51 \%$,

completed $30 \%$

Median follow- rash, anorexia, nausea $4 \%$, proctitis $2 \%$ obstruction, thrombosis

up 41 months: 5 Grade 4: DVT, rectal pain, anaemia 4\%; embolism $2 \%$

year OS $80 \%$, DFS leukopenia, neutropenia, fatigue,

naurther surgery

T-DS 83\%

Median follow-

up 34 months: DF

$6 \%$, LRF $0 \%$

Estimated 3 year abdominal pain $2 \%$

Grade 5: Aspiration 2\%, death related

to cancer $2 \%$

weight loss, hypertension, pelvic $3 \%$

$1 \%$ further surgery

$95.7 \%$ resection all $\mathrm{R0}, 68.1 \%$ SSS overall $36.2 \%$ cound healing problems $9.8 \%$, bleeding $2.8 \%$; GI perforation, grade 4 ileus $1.4 \%$

$91 \%$ resected, $88 \%$ R0

neutropenia $16 \%$; rectal pain,
leukopenia, fatigue $13 \%$, diarrhoea $11 \%$, Wound infection $16 \%$, fascial

leukopenia, fatigue $13 \%$, diarrhoea $11 \%$, Wound infection $16 \%$, fascial

Grade 3: hypertension 6\% (DL2)

Late complications $47 \%$. Wound infection $47 \%$, wound/fascial

dehiscence $24 \%$, bowel

obstruction/ileus $10 \%$, intraabdominal abscess $4 \%$, AL $2 \%$

$100 \%$ resected, $61 \%$ SSS

Grade 3 postoperative

complications: ileus, small bowel obstruction with pelvic abscess, cellulitis $6 \%$ 


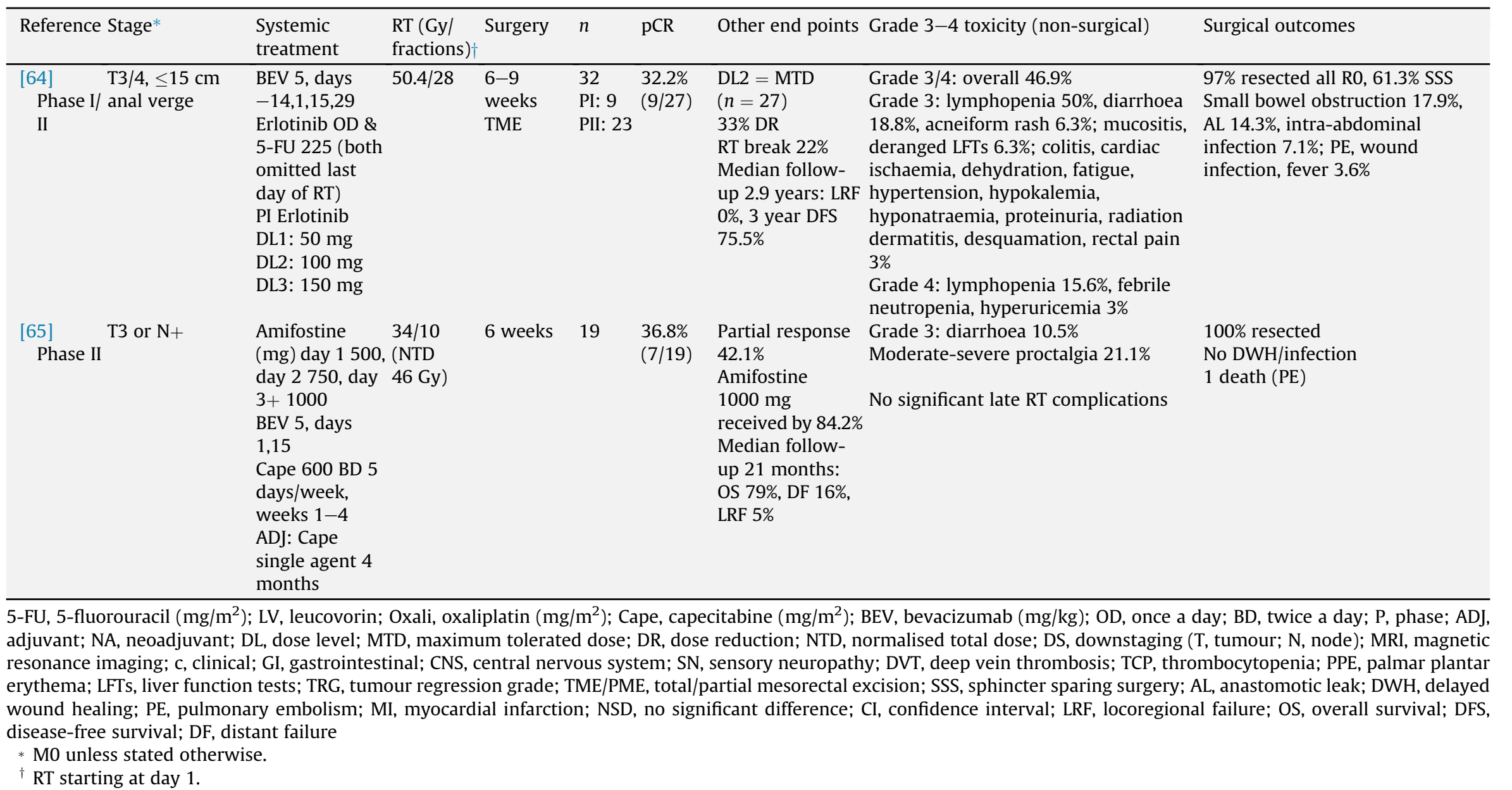


and clinical data have suggested that these combinations may have a synergistic effect; for example, bevacizumab enhances tumour blood flow and reduces tumour interstitial pressure and hypoxia $[40,41]$, potentially improving the activity of both chemotherapy and radiation.

\section{Biological Agents}

\section{Bevacizumab}

Bevacizumab, a humanised monoclonal antibody against vascular endothelial growth factor (VEGF), has been evaluated as a potential radiosensitiser when added to current CRT protocols. Proangiogenic factors such as VEGF can lead to radio-resistance, which theoretically could be reversed by such agents as bevacizumab [42].

Several single-arm phase II trials have assessed the efficacy and safety of the addition of bevacizumab to standard CRT, many of which have been reviewed elsewhere $[43,44]$ and are summarised in Table 3. Study size ranged from eight to 90 patients and of those completed, pCR ranged from 7.5 to $36.8 \%$. When reported, tumour downstaging was seen in $34.9-100 \%$ of patients $[45,46,54,55]$. In a variation to standard treatment, Koukourakis et al. [65] added bevacizumab to hypofractionated radiotherapy. Using a historical cohort of patients who had undergone hypofractionated radiotherapy [66], the authors showed that the pCR for bevacizumab cohort was $36.8 \%$ compared with $21.4 \%$ for the previous study, although this was not statistically significant. The only randomised study to date recently published results [55]. Ninety patients received radiotherapy with concurrent capecitabine and bevacizumab $5 \mathrm{mg} / \mathrm{kg}$ once every 2 weeks, or the same schedule without bevacizumab. The patients receiving bevacizumab had a higher pCR rate, but this was not statistically significant (16\% versus $11 \%$, $P=0.54$ ) and the predefined efficacy end point was not met. However, those receiving bevacizumab were more likely to achieve tumour downstaging (59\% versus 39\% $\mathrm{B} ; P=0.04$ ).

One interesting study assessed bevacizumab as an adjuvant or neoadjuvant therapy in a non-randomised manner [50]. This trial was designed as a single-arm adjuvant trial. However, because many physicians preferred a preoperative option, a second arm was added. Thirty-five patients had neoadjuvant CRT and 31 had CRT after surgery. The 1 year DFS rates were $85 \%$ (95\% confidence interval $67-93 \%$ ) and $97 \%$ (95\% confidence interval $79-100 \%$ ), respectively, with a pCR of $29 \%$ achieved in the neoadjuvant cohort. Severe bevacizumab-related toxicity occurred in $12 \%$ of patients (both cohorts) but was manageable and reversible. Overall, most trials have concluded that toxicities from CRT with bevacizumab were expected and manageable. A direct comparison in the RCT by Salazar et al. [55] found no statistical difference in preoperative toxicities between therapy with and without bevacizumab. However, two single-arm studies were terminated early due to excessive toxicity $[53,58]$.

Some trials have incorporated bevacizumab into a combination regimen (Table 3) of fluoropyrimidine, oxaliplatin and neoadjuvant radiotherapy. Tumour downstaging was seen in $44.9-81.9 \%$ and postoperative complications affected $18.3-63 \%$ of patients, where reported. However, the single-arm nature of these trials means that it is difficult to determine whether these are complications of oxaliplatin, bevacizumab or both. Within the phase II trials, pCR rates ranged from $16.6 \%$ to $18.4 \%$ in line with the previously described studies and similar to historic CRT trials without bevacizumab.

In conclusion, there is no definite evidence for which, if any, regimen is most appropriate to combine bevacizumab with standard neoadjuvant CRT and the addition of bevacizumab to CRT is therefore not currently recommended outside of clinical trials.

\section{Anti-epidermal Growth Factor Receptor}

The epidermal growth factor receptor (EGFR) is recognised as an important factor in colorectal cancer (CRC) initiation and progression. Elevated EGFR expression and activity frequently correlate with tumor resistance to radiotherapy, thought to be related to the activation of cell survival and proliferation pathways [67].

With the successful combination of cetuximab with radiotherapy in head and neck squamous cell carcinoma [68], there has been interest in its use as a radiosensitiser for other cancers. Cetuximab is already used routinely in the setting of metastatic CRC in patients with KRAS wild-type disease. Table 4 details clinical trials involving the addition of cetuximab to standard CRT with fluoropyrimidines. This regimen seems to be largely tolerable, with expected associated toxicities including rash, diarrhoea, mucositis and allergic reaction. Surgical toxicities where reported were not significantly increased $[70,74,75]$. However, an improvement in $\mathrm{PCR}$, which ranged from 0 to $28.3 \%$, has not been shown to date, even where KRAS status is taken into account. Two RCTs $[74,76]$ retrospectively analysed KRAS status and in the wild-type group found no difference in pCR with and without cetuximab. In the EXPERT-C trial [74], pCR rates of $11 \%$ versus $7 \%(P=0.71)$, respectively, were obtained, although of note, KRAS status was unavailable for eight patients who achieved pCR. However, there was evidence of increased response rates in the wild-type group who received cetuximab, $77.8 \%$ versus $53.8 \%(P=0.03)$. In a pooled analysis of two phase II studies of CRT, one with irinotecan [91] and the other irinotecan and cetuximab [80], Kim et al. [92] found that pathological response, postoperative pathological stage and DFS did not differ significantly. This lack of difference in outcomes remained even among KRAS wild-type participants and was corroborated by Kripp et al. [93], who compared two trials of CAPIRI $[13,94]$ with one adding cetuximab [79]. Sun et al. [75] in a single-arm trial also assessed KRAS status after treatment and noted no significant difference in PCR rates between wild-type and mutant, but did find higher levels of downstaging in the former, $86.4 \%$ versus $57.9 \%(P=0.02)$. Numbers in this trial were small and the difference in downstaging was not confirmed by Eisterer et al. [77] or Bertolini et al. [72]. In terms of survival data, Sun et al. [75] 
Table 4

Clinical trials of anti-epidermal growth factor receptor (EGFR) agents with neoadjuvant chemoradiotherapy (CRT) for rectal cancer

\begin{tabular}{|c|c|c|c|c|c|c|c|}
\hline \multicolumn{2}{|c|}{ Reference Disease stage* } & Systemic treatment $\left(\mathrm{mg} / \mathrm{m}^{2}\right)$ & $\begin{array}{l}\text { RT } \\
(\mathrm{Gy}) \dagger\end{array}$ & $n$ & $\mathrm{pCR}$ & Other end points & Grade $3-4$ toxicity (non-surgical) \\
\hline \multicolumn{8}{|c|}{ Cetuximab with neoadjuvant CRT phase I trial } \\
\hline [69] & $\begin{array}{l}\text { T3-4, locally } \\
\text { recurrent }\end{array}$ & $\begin{array}{l}\text { Cetux weeks } 1-10 \\
5 \text {-FU 225/day }\end{array}$ & 50.4 & 20 & $\begin{array}{l}12 \% \\
(2 / 17)\end{array}$ & $100 \%$ R0 & $\begin{array}{l}\text { Diarrhoea } 10 \% \text {, acneiform rash } 15 \% \text {; } \\
\text { stomatitis, radiation dermatitis, } \\
\text { transaminitis } 5 \%\end{array}$ \\
\hline \multicolumn{8}{|c|}{ Cetuximab with neoadjuvant CRT phase I/II trials } \\
\hline [70] & T3-4 \&/or N1-2 & $\begin{array}{l}\text { Cetux weeks }-1-10 \\
\text { Cape BD daily } \\
\text { DL1: } 650 \\
\text { DL2: } 825\end{array}$ & 45 & 40 & $\begin{array}{l}5 \% \\
(2 / 37)\end{array}$ & $\begin{array}{l}\text { Dworak TRG3 27\% } \\
\text { DS 38\% } \\
92.5 \% \text { resected, } 100 \% \text { R0, } 75.7 \% \text { SSS }\end{array}$ & $\begin{array}{l}\text { Grade 3: diarrhoea } 15 \% \text {, allergy Cetux } \\
3 \% \\
\text { Grade 4: MI, PE, fatal sepsis } 3 \%\end{array}$ \\
\hline [71] & $\mathrm{T} 3 / 4 \mathrm{NO} / 1$ & $\begin{array}{l}\text { Cetux weekly } \\
\text { 5-FU days } 1-5,29-33 \\
\text { DL1: } 750 \\
\text { DL2: } 1000\end{array}$ & 50.4 & 20 & $\begin{array}{l}5.5 \% \\
(1 / 19)\end{array}$ & $\begin{array}{l}\text { MTD 5-FU } 750 \mathrm{mg} / \mathrm{m}^{2} \\
90 \% \text { resected, } 100 \% \mathrm{R} 0,67 \% \text { SSS }\end{array}$ & $\begin{array}{l}\text { Allergic reaction Cetux } 5 \% \\
\text { DL2 DLTs: grade } 3 \text { diarrhoea, TCP } \\
\text { Death } 20 \% \text { ( } 3 / 4 \text { due to metastatic } \\
\text { disease progression) }\end{array}$ \\
\hline \multicolumn{8}{|c|}{ Cetuximab with neoadjuvant CRT phase II trials } \\
\hline [72] & $\mathrm{T} 3 / 4 \mathrm{~N} 0 / 1$ & $\begin{array}{l}\text { Cetux weeks }-3-5 / 6 \\
5 \text {-FU } 225\end{array}$ & $\begin{array}{l}50- \\
50.4\end{array}$ & 40 & $\begin{array}{l}8 \% \\
(3 / 38)\end{array}$ & $\begin{array}{l}\text { Dworak TRG } 318 \% \\
\text { DS } 60.5 \% \\
95 \% \text { resected }\end{array}$ & $\begin{array}{l}\text { Grade } 3-4 \text { : skin rash, hypersensitivity } \\
\text { reaction, diarrhoea } 7.5 \% \text {; stomatitis, } \\
\text { liver enzyme, febrile neutropenia } 2.5 \%\end{array}$ \\
\hline [73] & II/III & $\begin{array}{l}\text { Cetux weeks }-1-5 \\
\text { Cape } 1250 \text { BD week }-3 \text { and }-2 \\
\text { then } 825 \text { BD weeks } 1-5 \\
\text { ADJ: Cape x3 cycles }\end{array}$ & 45 & 40 & $\begin{array}{l}8 \% \\
(3 / 37)\end{array}$ & $\begin{array}{l}\text { 7\% withdrew trial CRT due to cardiac } \\
\text { ischaemia/chest pain } \\
\text { Dworak TRG3 19\% } \\
\text { DS } 73 \% \\
\text { R0 } 97 \%\end{array}$ & $\begin{array}{l}\text { Grade 3: radiodermatitis } 16 \% \text {, } \\
\text { diarrhoea } 11 \% \text {, hypersensitivity } 5 \% \\
\text { (Cetux discontinued after \#1 in } 11 \% \text { ); } \\
\text { hepatotoxicity, infection, anorexia } 3 \%\end{array}$ \\
\hline [74] & $\begin{array}{l}\text { High risk operable: } \\
<1 \mathrm{~mm} \text { CRM, } \\
\text { Low T3 or T4, EMVI, } \\
\text { Extramural } \\
\text { extension }>4 \mathrm{~mm}\end{array}$ & $\begin{array}{l}\text { RCT } \\
\text { A: NA CAPOX x } 4 \\
\text { Cape } 825 \text { BD daily + RT } \\
\text { B: above + Cetux weekly }\end{array}$ & 50.4 & $\begin{array}{l}165 \\
\mathrm{~A}: 81 \\
\mathrm{~B}: 84\end{array}$ & $\begin{array}{l}\text { KRAS/BRAF WT } \\
\text { A: } 7 \%(3 / 44) \\
\text { B: } 11 \%(5 / 46) \\
P=0.71\end{array}$ & $\begin{array}{l}\text { Median follow-up } 32 \text { versus } 37 \\
\text { months. All: NSD PFS, OS, } 91 \% / 94 \% \\
\text { resected, } 92 \% / 96 \% \text { R0, } 73 \% / 72 \% \text { SSS. } \\
\text { WT: radiologic response after CRT } \\
75 \% / 93 \%(P=0.028) \text {, OS benefit with } \\
\text { Cetux (HR } 0.27, P=0.034) \text {; NSD R0, } \\
\text { SSS }\end{array}$ & $\begin{array}{l}\text { Grade } 3-5 \text { : diarrhoea } 1 \% / 10 \% \text {, rash } \\
0 \% / 9 \% \text {, PPE } 1 \% / 4 \% \\
2 \text { peri-operative deaths (arm A) }\end{array}$ \\
\hline [75] & $\begin{array}{l}\mathrm{T} 3 / \mathrm{T} 4 \text { (R0/1 } \\
\text { achievable) }\end{array}$ & $\begin{array}{l}\text { Cetux weeks } 1-5 \\
\text { Cape } 1250 \text { BD week } 1,850 \text { BD } \\
\text { weeks } 2-5\end{array}$ & 45 & 63 & $\begin{array}{l}12.7 \%(8 / 63) \\
\text { KRAS: } \\
\text { MT } 10.5 \%(2 / 19) \\
\text { WT } 13.6 \%(6 / 44) \\
P=1.0\end{array}$ & $\begin{array}{l}\text { All: DS } 77.8 \% \\
3 \text { year DFS } 76.2 \% \text {, OS } 81 \% \\
\text { DS KRAS WT } 86.4 \% / \text { MT } 57.9 \% \\
(P=0.02), 3 \text { year DFS } 76.7 \% / 75 \%, 3 \\
\text { year OS } 81.4 \% / 80 \%(N S D)\end{array}$ & $\begin{array}{l}\text { Grade } 3 \text { : radiodermatitis } 16 \% \text {; } \\
\text { diarrhoea, acneiform rash } 6 \% \text {; dry } \\
\text { skin, infection } 3 \%\end{array}$ \\
\hline [76] & $\mathrm{T} 3 / 4$ or $\mathrm{N}+$ & 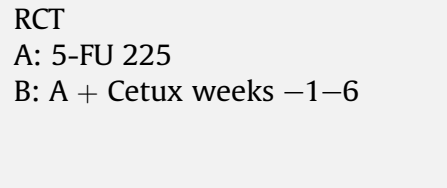 & 50.4 & $\begin{array}{l}139 \\
\text { A: } \\
69 \\
\text { B: } \\
70\end{array}$ & $\begin{array}{l}\text { A: } 28.3 \%(17 / 60) \\
\text { B: } 26.6 \%(17 / 64) \\
\text { NSD }\end{array}$ & $\begin{array}{l}5 \text { year PFS } 61 \% / 65 \% \text {, OS } 66 / 83 \%, \text { LRF } 3 / \\
4 \%\end{array}$ & $\begin{array}{l}\text { Diarrhoea } 16 \% / 22 \% \text {, rash } 0 \% / 12 \% \text {, } \\
\text { mucositis } 5 \% / 6 \% \text {, dehydration } 5 \% / 8 \%\end{array}$ \\
\hline [77] & $\begin{array}{l}\text { cT3/4 potentially } \\
\text { resectable }\end{array}$ & $\begin{array}{l}\text { Cetux weeks } 1-5 \\
\text { Cape } 825 \text { BD } 5 \text { days/week } \\
\text { weeks } 1-4\end{array}$ & 45 & 31 & $0 \%(0 / 28)$ & $\begin{array}{l}\text { R0 96\%, } \\
\text { DS } 46 \% \\
\text { KRAS status in } 25 \text { patients ( } 56 \% \text { WT, } \\
44 \% \text { MT) showed no correlation with } \\
\text { DS }\end{array}$ & $\begin{array}{l}\text { Grade 3: diarrhoea } 10 \% \text {, rash } 6 \% \text {, rectal } \\
\text { itching/pain } 3 \% \\
\text { Grade 4: diarrhoea } 6 \% \text {, weight loss } 3 \%\end{array}$ \\
\hline
\end{tabular}


Cetuximab and irinotecan with neoadjuvant CRT phase I or II trials

\begin{tabular}{|c|c|c|c|c|c|}
\hline $\begin{array}{l}{[78]} \\
\text { Phase I }\end{array}$ & $\begin{array}{l}\text { Cetux weeks } 1-5 \\
\text { Irino weekly weeks } 1-5 \\
\text { Cape BD } \\
3 \text { DLs: } \\
\text { 1. Irino } 40 \text {, Cape } 400 \\
\text { 2. Irino } 40 \text {, Cape } 500 \\
\text { 3. Irino } 50 \text {, Cape } 500\end{array}$ & 50.4 & 20 & $26 \%(5 / 19)$ & $\begin{array}{l}\text { DL2 accepted } \\
\text { R0 } 95 \% \\
\text { T-DS } 42 \% \\
\text { N-DS } 67 \%\end{array}$ \\
\hline $\begin{array}{l}\text { [79] }{ }^{\mathrm{cT}} 3-4 / \mathrm{N}+ \\
\text { Phase II }\end{array}$ & $\begin{array}{l}\text { Cetux weeks } 1-5 \\
\text { Irino } 40 / \text { week weeks } 1-5 \\
\text { Cape } 500 \text { BD }\end{array}$ & 50.4 & 50 & $8 \%(4 / 50)$ & $\begin{array}{l}\text { All R0 } \\
68 \% \text { moderate to good tumour } \\
\text { regression }\end{array}$ \\
\hline $\begin{array}{l}\text { [80] mrT3 or resectable } \\
\text { Phase II } 44 \text { or } \mathrm{N}+\end{array}$ & $\begin{array}{l}\text { Cetux weeks }-1-5 \\
\text { Irino } 40 / \text { week weeks } 1-5 \\
\text { Cape } 825 \text { BD } 5 \text { days/week } \\
\text { ADJ: 5-FU/LV }\end{array}$ & 50.4 & 39 & $23.1 \%(9 / 39)$ & $\begin{array}{l}\text { T-DS } 53.8 \% \\
\text { All resections R0, SSS } 79.5 \% \\
3 \text { year DFS } 80 \% \text {, OS } 94.7 \% \\
\text { KRAS MT } 13.2 \%(5 / 38) . \text { NSD in TRG } \\
\text { DFS versus WT }\end{array}$ \\
\hline $\begin{array}{l}{[81,82] \quad c T 2-4 / \mathrm{NO}-2} \\
\text { Phase II }\end{array}$ & $\begin{array}{l}\text { Cetux weeks }-1-5 \\
\text { Irino } 60 / \text { week weeks } 1-4 \\
\text { Cape } 650 \text { BD } 5 \text { days/week }\end{array}$ & 45 & 82 & $18 \%(14 / 76)$ & $\begin{array}{l}\text { R0 } 89 \% \\
\text { T-DS } 51 \% \\
\text { N-DS } 78 \% \\
4 \text { cCR did not have surgery }\end{array}$ \\
\hline $\begin{array}{l}\text { Cetuximab and oxaliplatin wit } \\
\text { [83] uT3-4/N1 } \\
\text { Phase I }\end{array}$ & $\begin{array}{l}\text { neoadjuvant CRT phase I or II trial } \\
\text { Cetux weeks } 1-9 \\
\text { Oxali } 100 \text { days } 2,23 \\
\text { Cape } 800 \text { BD } 5 \text { days/week } \\
\text { weeks } 1-6\end{array}$ & s 50.4 & 23 & $17 \%(4 / 23)$ & DS 57\% \\
\hline 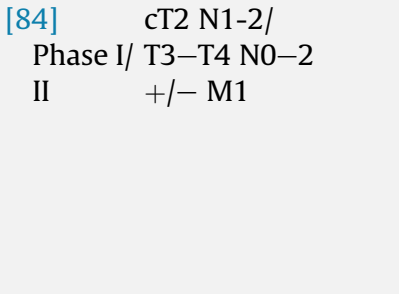 & $\begin{array}{l}\text { PI: } \\
\text { Cape BD weeks } 1-2,4-5 \\
\text { DLs 500/650/825, } \\
\text { Cetux weeks }-1-6 \text {, } \\
\text { Oxali 50/week weeks } 1-5 \\
\text { PII: } \\
\text { Cape DL3 } \\
\text { Cetux/Oxali as above }\end{array}$ & 50.4 & $\begin{array}{l}\text { PI: } \\
13 \\
\text { PII: } \\
48\end{array}$ & $9 \%(4 / 45)$ & $\begin{array}{l}\text { PII: } 11 \% \mathrm{M} 1 \\
\text { RT given as prescribed } 92 \% \\
\text { T-DS } 47 \% \\
\text { N-DS } 58 \% \\
\text { Resected } 95 \% \text {, R0 93\% }\end{array}$ \\
\hline $\begin{array}{l}\text { [85] T3-4Nx/T2N+M1 } \\
\text { Phase II }\end{array}$ & $\begin{array}{l}\text { Cetux (regimen not stated) } \\
\text { Oxali 50/week weeks } 1-5 \\
\text { Cape } 800 \text { BD daily } \\
\text { ADJ: FOLFOX/Cetux }\end{array}$ & 50 & 19 & Not given & $\begin{array}{l}63 \% \text { CRT completed } \\
68 \% \text { resected }\end{array}$ \\
\hline $\begin{array}{l}{[86] \text { LARC }} \\
\text { Phase II } K R A S \text { WT }\end{array}$ & $\begin{array}{l}\text { NA: } 2 \times 21 \text { day cycles Cape } 1000 \\
\text { BD days } 1-14 \text {, Oxali } 130 \& \text { BEV } \\
7.5 \mathrm{mg} / \mathrm{kg} \text { day } 1 \\
\text { CRT: } \\
\text { Cetux } 400 \text { 2-weekly } \\
\text { Cape } 650 \text { BD daily }\end{array}$ & 50.4 & 10 & $50 \%(2 / 4)$ & \\
\hline
\end{tabular}

DL2 (10 patients)

Grade 3: diarrhoea $20 \%$, transaminitis $10 \%$

Grade 3: diarrhoea 30\%, transaminitis $10 \%$, acne-like rash $6 \%$, abdominal pain $4 \%$; anaemia, leukopenia, $N \& V 2 \%$ Grade 4: $2 \%$ leukopenia

Grade 3: leukopenia 7.7\%;

neutropenia, diarrhoea $5.1 \%$; anaemia fatigue, rash, ileus $2.6 \%$

Grade 4: leukopenia 2.6\%

Grade 3: diarrhoea 25\%, acne-like rash $9 \%$, fatigue $8 \%$; thrombotic event

febrile neutropenia $1 \%$

Grade 4: thrombotic event 6\%, febrile neutropenia $1 \%$

Oxali stopped after 10 patients (radiosensitising toxicities)

3 withdrew: 2 EGFR skin toxicity, 1

RT-induced ileus

PII: death multi-organ failure (DPD deficient) $2 \%$

Grade 3: diarrhoea $17 \%$, radiation dermatitis $8 \%$; transaminitis, infection/fever, SN 6\%; leukopenia, $\mathrm{N} \& \mathrm{~V}$, acne-like rash $4 \%$; TCP, ileus, cardiac toxicity, allergic reaction/ hypersensitivity, proctitis $2 \%$

Grade 3: diarrhoea 26\%

Stopped at interim analysis (toxicity/ lack efficacy)

Available for 8 patients Grade 3: proctitis 13\% 


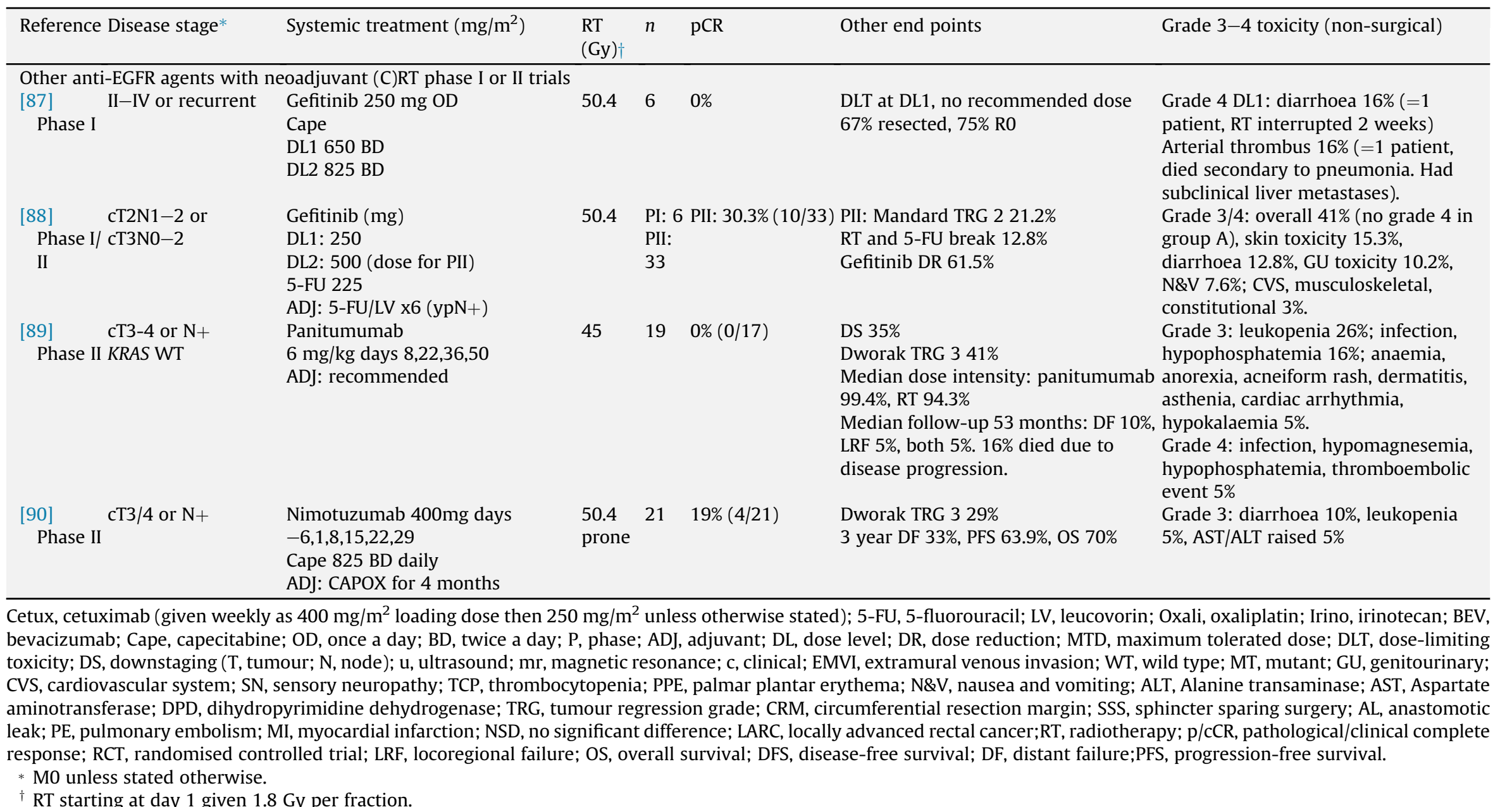

${ }^{\dagger}$ RT starting at day 1 given 1.8 Gy per fraction. 
Table 5

Newer agents in combination with radiotherapy (RT) in early phase trials

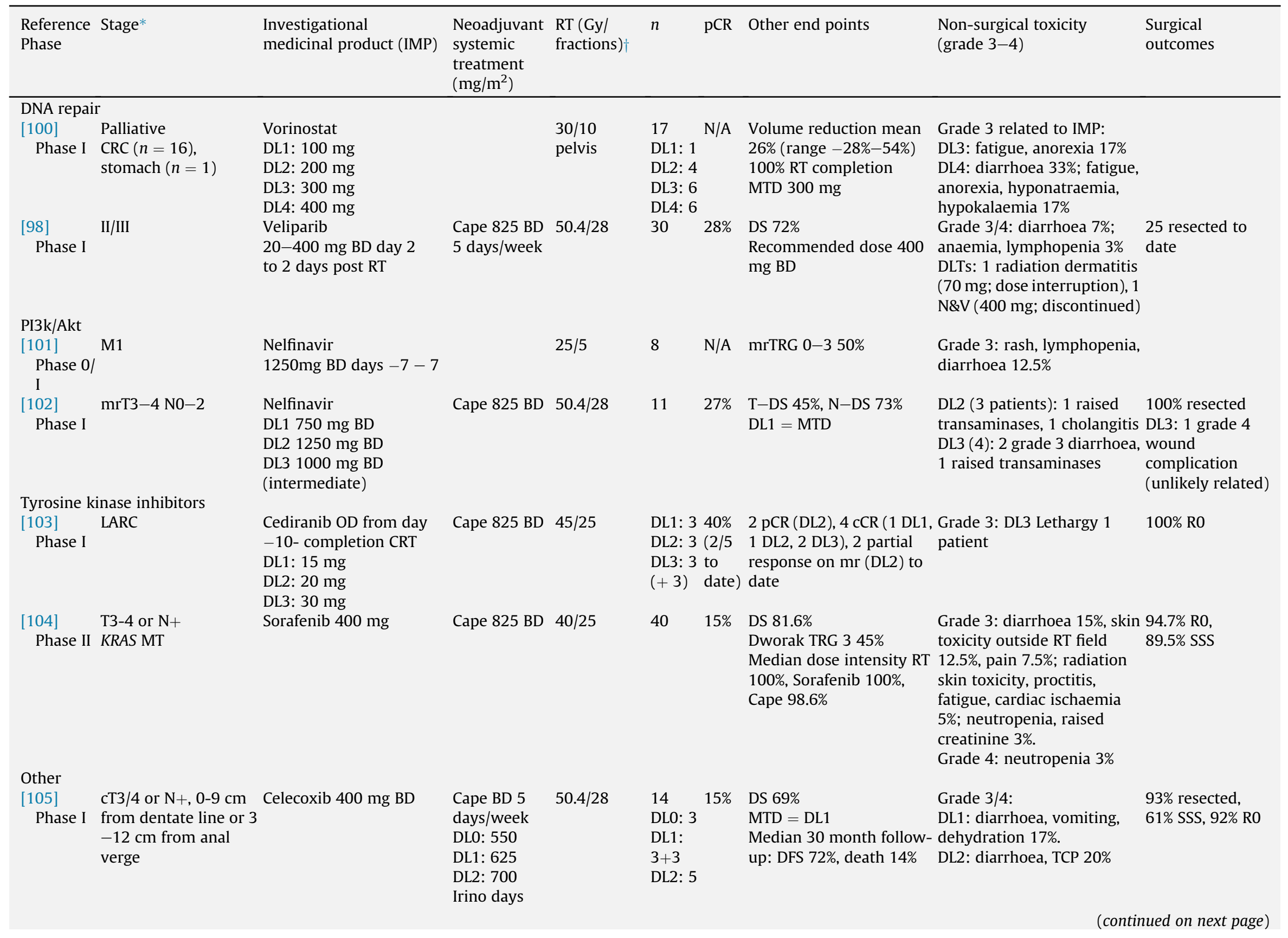




\begin{tabular}{|c|c|c|c|c|c|c|c|c|c|}
\hline $\begin{array}{l}\text { Reference } \\
\text { Phase }\end{array}$ & Stage* & $\begin{array}{l}\text { Investigational } \\
\text { medicinal product (IMP) }\end{array}$ & $\begin{array}{l}\text { Neoadjuvant } \\
\text { systemic } \\
\text { treatment } \\
\left(\mathrm{mg} / \mathrm{m}^{2}\right)\end{array}$ & $\begin{array}{l}\mathrm{RT}(\mathrm{Gy} / \\
\text { fractions }) \dagger\end{array}$ & $n$ & $\mathrm{pCR}$ & Other end points & $\begin{array}{l}\text { Non-surgical toxicity } \\
\text { (grade } 3-4 \text { ) }\end{array}$ & $\begin{array}{l}\text { Surgical } \\
\text { outcomes }\end{array}$ \\
\hline $\begin{array}{l}{[106]} \\
\text { Phase I }\end{array}$ & $\mathrm{uT} 3 / 4$ or $\mathrm{N}+\mathrm{MO} / 1$ & $\begin{array}{l}\text { Bortezomib }\left(\mathrm{mg} / \mathrm{m}^{2}\right) \\
\text { DL1: } 0.7 \\
\text { DL2: } 1.0 \\
\text { DL3: } 1.3 \\
\text { DL4: } 1.5 \\
\text { Biweekly weeks } 1,2,4,5\end{array}$ & $\begin{array}{l}\text { 1,8,22,29 } \\
\text { DL0: } 30 \\
\text { DL1: } 35 \\
\text { DL2: } 40 \\
\text { 5-FU 225/day } \\
\text { from day } 2\end{array}$ & $50.4 / 28$ & $\begin{array}{l}9 \\
\text { DL1: } 3 \\
\text { DL2: } 6\end{array}$ & $11 \%$ & $\begin{array}{l}\text { Microscopic residual 11\% } \\
\text { MTD } 0.7 \mathrm{mg} / \mathrm{m}^{2}\end{array}$ & $\begin{array}{l}\text { Grade } 3 / 4 \text { : } \\
\text { DL1 chest pain, diarrhoea, } \\
\text { hyperglycaemia, } \\
\text { lymphopenia } 17 \% \text {. } \\
\text { DL2 diarrhoea } 67 \% \text {; } \\
\text { hypoalbuminemia, } \\
\text { hypokalaemia, ileus } 33 \%\end{array}$ & $100 \%$ resected \\
\hline $\begin{array}{l}{[107]} \\
\text { Phase I }\end{array}$ & $\begin{array}{l}\mathrm{T} 3 / 4 \text { or } \mathrm{N}+\text { or local } \\
\text { recurrence }\end{array}$ & $\begin{array}{l}\text { TNFerade }{ }^{\mathrm{TM}}(\mathrm{TNF}-\alpha) \\
4 \times 10^{10} \text { particle units } \\
\text { direct tumour injection } \\
\text { weekly } \times 5\end{array}$ & $\begin{array}{l}\text { Cape } 937.5 \\
\text { BD } 5 \text { days/ } \\
\text { week }\end{array}$ & $\begin{array}{l}45 / 25+5.4 \\
-9 \text { Gy boost }\end{array}$ & 9 & $22 \%$ & $\begin{array}{l}\text { Microscopic foci/no } \\
\text { residual } 78 \% \\
\text { Median follow-up } 41.6 \\
\text { months: } 1 \text { death } \\
\text { (metastases), } 2 \text { DF, } 2 \text { LRF } \\
\text { (1 also DF) }\end{array}$ & $\begin{array}{l}\text { Grade } 3 / 4 \text { : lymphopenia } \\
100 \% \text {; hypophosphatemia, } \\
\text { hyponatraemia } 50 \% \text {, } \\
\text { neutropenia } 38 \% \text {; anaemia, } \\
\text { diarrhoea, small bowel } \\
\text { obstruction, LFT } \\
\text { abnormalities } 25 \% \text {, enteritis } \\
13 \% \\
1 \text { patient stopped CRT }\end{array}$ & $89 \%$ SSS \\
\hline $\begin{array}{l}\text { [108] } \\
\text { Phase I }\end{array}$ & II/III & $\begin{array}{l}\text { Nitroglycerin transdermal } \\
\text { patches }(\mathrm{mg} / \mathrm{h}) \\
\text { DL1 } 0.2 \\
\text { DL2 } 0.4 \\
\text { DL3 } 0.6\end{array}$ & 5-FU 225/day & $\begin{array}{l}45-50 / \\
25-28\end{array}$ & 13 & $17 \%$ & $\begin{array}{l}\text { No DLTs } \\
\text { 8\% RT break }\end{array}$ & $\begin{array}{l}\text { Grade 3: lymphopenia } 31 \% \text {; } \\
\text { diarrhoea, headache, } \\
\text { mucositis } 8 \% \text {. }\end{array}$ & $\begin{array}{l}92 \% \text { resected, } \\
67 \% \text { SSS }\end{array}$ \\
\hline
\end{tabular}

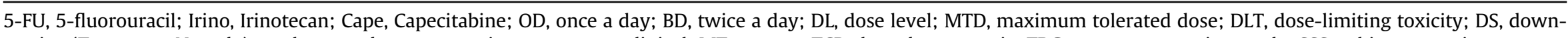

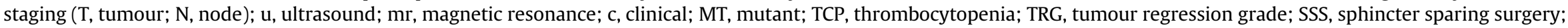
LARC, locally advanced rectal cancer; CRC, colorectal cancer; CRT, chemoradiotherapy; pCR, pathological complete response; N\&V, nausea and vomiting; DFS, disease-free survival;

$\mathrm{DF}$, distant failure; LRF, locoregional failure;

* M0 unless stated otherwise.

${ }^{\dagger}$ RT starting at day 1. 
showed no difference in 3 year DFS or overall survival between KRAS wild-type and mutant groups. Updated analysis of the EXPERT-C data by Sclafani et al. [95] showed no significant difference in 5 year overall survival at $83.8 \%$ versus $70.0 \%(P=0.20)$ with and without cetuximab in KRAS wildtype.

Table 4 lists trials in which cetuximab was added to combination chemotherapy regimens including oxaliplatin or irinotecan with fluoropyrimidine and radiotherapy. Combining three systemic agents seems to increase toxicity and two trials using oxaliplatin $[83,85]$ were altered or closed early for this reason. pCR varied between 8 and 50\% and downstaging rates did not seem to be different from those who received just two systemic agents.

Other EGFR inhibitors are now under evaluation with CRT in rectal cancer, including gefitinib, panitumumab and nimotuzumab. Erlotinib has also been studied in combination with bevacizumab [64] (Table 3). pCR rates range between 0 and $30 \%$ with downstaging rates of $21-35 \%$. Jin et al. [90] had a high proportion of KRAS wild-type patients at $86 \%$, which they postulate may have contributed to the pCR rate of $19 \%$ with nimotuzumab. However, in the study with panitumumab [89], which only included participants with KRAS wild-type, the pCR rate was $0 \%$. The trials concluded that toxicity was generally manageable. The use of cetuximab or other EGFR inhibitors in combination with CRT therefore seems to be feasible, but there is little evidence of improvement in clinically important outcomes in early phase trials. Relevant and robust biomarkers of efficacy and toxicity will be required for further assessment of EGFR inhibitors in larger randomised trials.

\section{Newer Agents}

In recent years, advances in sequencing technology have seen a rapid expansion in our knowledge of the range of genetic mutations found in CRC and characterisation of the associated phenotypes. This knowledge has suggested potential new targets for therapy, some of which are relevant to radiosensitisation.

There has been increasing interest in novel radiosensitisers that prevent repair of DNA damage caused by ionising radiotherapy. Pre-clinical data have found that poly(ADP-ribose) polymerase (PARP) inhibitors prevent PARP from facilitating repair of single-strand breaks, leading to inability of DNA replication in S-phase and the formation of lethal double-strand breaks [96]. In CRC with microsatellite instability, there is evidence of a deficient double-strand break repair system related to mutations in coding microsatellites. This deficiency may be exploited using PARP inhibitors in a manner similar to their successful use in BRCA1-mutant breast cancers [97]. Czito et al. [98] recently published early results of a phase I trial of the oral PARP inhibitor, veliparib, in combination with CRT for rectal cancer. pCR was $28 \%$, and $72 \%$ of 25 patients treated had tumour downstaging. Two dose-limiting toxicities (DLTs) were experienced and a recommended dose for phase II trials of $400 \mathrm{mg} \mathrm{BD}$ was advocated by the investigators. The histone deacetylase inhibitor, vorinostat, acts as a radiosensitiser by downregulating proteins involved in the signalling and repair of radiation-induced double-strand breaks [99]. Ree et al. [100] added vorinostat to palliative pelvic radiotherapy in 14 patients, most with CRC. The mean reduction in tumour volume was $26 \%$ (range $-28 \%-54 \%$ ) but toxicity was higher than with singleagent treatment (Table 5).

Genetic alterations in the RAS-MAPK and PI3-K/AKT pathways are common in CRC [109]. The latter is often overactivated in solid tumours and is an important tumour cell survival pathway with emerging evidence of involvement in hypoxia-related treatment resistance [110]. Nelfinavir, an AKT-inhibitor in the PI3-K pathway, is a radiosensitiser that has been shown in preclinical models to reduce tumour angiogenesis and hypoxia [111]. In an early phase study of patients undergoing short-course palliative pelvic radiotherapy, Hill et al. [101] found good mrTRG 0-3 in $50 \%$ of participants and the addition of nelfinavir was generally well tolerated. Buijsen et al. [102] undertook a dose escalation study of nelfinavir in patients receiving CRT with capecitabine and recorded a pCR rate of $27 \%$ in 12 patients. DLTs included grade 3 cholangitis (one), transaminase elevation (two) and grade 3 diarrhoea (two). By contrast, no DLT related to the study medication was found in studies evaluating nelfinavir for CRT in pancreatic and lung cancer [112,113]. The authors concluded that the combination is therefore feasible but that safety and efficacy, particularly in combination with pelvic radiotherapy, requires further assessment.

The addition of bevacizumab to CRT has had limited success in improving patient outcomes, as discussed previously. Other agents targeting this pathway are under investigation, including cediranib, a VEGF receptor Tyrosine kinase inhibitor (TKI), which has shown good tolerability and promising outcomes in terms of response ( $\mathrm{pCR}=25 \%$, $n=8$ ) [103]. The same study is also evaluating the MEK inhibitor, selumetinib, and final results are awaited. Van Moos et al. [104] combined sorafenib, a multikinase inhibitor of pathways including RAF/MEK/ERK and VEGF-R, with capecitabine and radiotherapy in a phase II study involving 40 patients with KRAS mutant disease. With pCR rates of $15 \%$, downstaging of $81.6 \%$ and acceptable toxicity levels, they concluded that this regimen also warrants further investigation.

Bortezomib is a proteasome inhibitor that results in cell cycle redistribution and inhibition of transcription factors such as nuclear factor- $\kappa \mathrm{B}(\mathrm{NF}-\kappa \mathrm{B})$, which promotes cell survival and proliferation and has been shown to be activated in CRC models in response to chemotherapy and radiation [114]. However, O'Neill et al. [106] saw mixed results on analysis of NF- $\kappa B$ gene expression in a clinical study of bortezomib with CRT. The trial was unable to recommend a biologically meaningful dose due to toxicity, similar to Edelman et al. [115] in lung cancer CRT, where delayed toxicity was a concern. Other phase I trials have analysed the effects of the cyclooxygenase-2 (COX-2) inhibitor celecoxib [105] and nitric oxide donor nitroglycerin [108] in combination with CRT, both of which showed potential as 
radiosensitisers in preclinical models and, the authors suggested, warrant further investigation following phase I studies. Another early phase trial utilised intratumoral injections of tumour necrosis factor-alpha (TNF- $\alpha$ ), a soluble cytokine that mediates cellular immune response, alongside CRT in rectal cancer and found that its use was feasible in this setting. Increased toxicity attributed to the TNF- $\alpha$ included fever, rigors and fatigue, all of which were low grade [115].

Increasing interest in the use of anti-cancer immunotherapy may indicate one future direction for novel radiosensitiser research. Radiotherapy has been noted to contribute to anti-tumour immunity through a variety of mechanisms, including release of tumour antigen through cell death, triggering of proinflammatory signals, which leads to activation of tumour-specific T-cells, and effects on the tumour microenvironment, which enhance T-cell infiltration [116]. In addition, a systemic anti-tumour immune response outside of the radiation field, termed the abscopal effect, has been described after radiotherapy. Pre-clinical models and early phase trials summarised in [117] have suggested a role for the promotion of T-cell priming with radiotherapy. The checkpoint inhibitors CTLA-4 and PD-1, which play a key role in the negative regulation of T-cell activity, are potential targets and blockade of the latter has been shown in animal models of CRC to enhance the therapeutic efficacy of radiotherapy [118]. Combining radiotherapy with other immunostimulatory agents may enhance their efficacy. The use of tumour neoantigens to stimulate immune response, for example through the creation of synthetic vaccines, has also been suggested as a novel treatment strategy with radiotherapy [116] and may be particularly relevant in cancers such as CRC, which have a high somatic mutation prevalence [119].

\section{Discussion}

Various explanations have been provided as to the limited improvement in outcomes seen with the addition of radiosensitising agents to standard CRT. It has been hypothesised that optimal radiotherapy with fluoropyrimidines already maximises the local response with little scope for increasing the radiosensitiser effect [30]. Systemic agents may target different phases of the cell cycle, and radiosensitisation may be partly dependent on cell cycle synchronisation of the tumour cell population. Given that fluoropyrimidines are S-phase specific and oxaliplatin is also mainly active in this phase, cell cycle delay or arrest may limit effectiveness and increase time for DNA repair. This may be of concern with agents such as cetuximab, which can lead to G1 or G2/M cell cycle arrest [120]. An understanding of the underlying biological mechanisms of novel radiosensitisers will be fundamental to future research in this area.

Toxicity is a significant consideration when combining radiosensitising agents with radiotherapy. There is evidence that delivery of radiotherapy and therefore treatment efficacy could be compromised due to the toxicity of additional therapies such as oxaliplatin [37]. Nonetheless, the importance of radiotherapy quality assurance should not be overlooked when investigating systemic agents in this context. For example, Bratland et al. [121] found that toxicity with vorinostat and pelvic radiotherapy was considerably higher than with the drug alone, but upon reanalysis of their data, radiation dose-volume effects were identified as a potential contributory factor. Toxicity has also limited the combination with CRT of higher than radiosensitising doses of chemotherapy, which had been postulated to provide a survival benefit through the treatment of systemic micrometastases [30].

In the case of biological agents, there is currently a paucity of direct comparison with placebo and limited ability to make accurate patient selection. Gollins et al. [82] in their study of CRT with cetuximab found retrospectively that in $22 \%$ of the participants where EGFR pathway mutation status was available, this differed between biopsy and surgical resection specimen, leading them to question the validity of using biopsy-derived mutation status to select treatment. Effective predictive biomarkers are required to select patients who would benefit most from targeted therapy. Cubillo et al. [122] have started to personalise CRT based on molecular markers with some success and this may indicate the direction of future research, in a similar vein to the molecular selection strategy seen in the FOCUS4 trial in metastatic CRC [123]. In addition, future work with biological therapies must also strive to further characterise the safety profile, given the mixed evidence of increased perioperative toxicity seen, particularly with antiangiogenesis agents.

Finally, choosing appropriate end points will be essential for any future research. Although pCR has been found to reflect good outcomes for that patient group [124,125], Kennecke [59] reported that central review changed pathological stage in six cases (16\%) in a trial of CRT with oxaliplatin and bevacizumab, highlighting a potential issue with this measure. Clinically relevant, robust outcomes will provide valuable evidence for any change to standard CRT regimens for locally advanced rectal cancer.

\section{Conclusions}

The addition of further cytotoxic agents to standard CRT has thus far provided largely disappointing results, with little improvement in outcomes, often at the expense of greater toxicity. With the advent of biological therapy, it was hoped that their use as radiosensitisers would provide significant synergistic effect without the side-effects of cytotoxic agents. However, in the case of bevacizumab and cetuximab, their addition to CRT with fluoropyrimidines has not proved successful so far. Debate exists as to whether the use of surrogate end points such as pCR adequately reflects long-term clinical outcomes, and which primary end point to use will remain an important consideration for future research. Nevertheless, the ability to tailor targeted therapy based upon a greater understanding of tumour genetic information, increasing 
availability of predictive biomarkers and a growing range of novel agents still has the potential to improve patient outcomes in the future.

\section{Conflict of Interest}

\author{
Charles Dearman is a freelance managing editor for \\ Elsevier.
}

\section{Acknowledgements}

We thank Elinor Hariss, librarian at The Knowledge Centre, Bodleian Health Care Libraries, University of Oxford, who assisted with the literature search. TAG and RAS are supported by the NIHR Oxford Biomedical Research Centre and the CRUK/EPSRC Oxford Cancer Imaging Centre (C5255/ A16466).We apologise to authors whose work has not been cited in this overview due to limits on word count.

\section{References}

[1] Krook JE, Moertel CG, Gunderson LL, et al. Effective surgical adjuvant therapy for high-risk rectal carcinoma. N Engl J Med 1991;324(11):709-715.

[2] Sauer R, Becker H, Hohenberger W, et al. Preoperative versus postoperative chemoradiotherapy for rectal cancer. $N$ Engl J Med 2004;351(17):1731-1740.

[3] Bosset JF, Calais G, Mineur L, et al. Enhanced tumorocidal effect of chemotherapy with preoperative radiotherapy for rectal cancer: preliminary results-EORTC 22921. J Clin Oncol 2005;23(24):5620-6567.

[4] National Institute for Health and Care Excellence. Colorectal cancer: the diagnosis and management of colorectal cancer (NICE clinical guideline 131) 2014.

[5] Glynne-Jones R, Mawdsley S, Pearce T, Buyse M. Alternative clinical end points in rectal cancer-are we getting closer? Ann Oncol 2006;17(8):1239-1248.

[6] Sargent DJ, Patiyil S, Yothers G, et al. End points for colon cancer adjuvant trials: observations and recommendations based on individual patient data from 20,898 patients enrolled onto 18 randomized trials from the ACCENT Group. J Clin Oncol 2007;25(29):4569-4574.

[7] Chen AY, Choy H, Rothenberg ML. DNA topoisomerase Itargeting drugs as radiation sensitizers. Oncology 1999;13(10 Suppl. 5):39-46.

[8] Illum H. Irinotecan and radiosensitization in rectal cancer. Anti-Cancer Drugs 2011;22(4):324-329.

[9] Iles S, Gollins S, Susnerwala S, et al. Irinotecan+5-fluorouracil with concomitant pre-operative radiotherapy in locally advanced non-resectable rectal cancer: a phase I/II study. Br J Cancer 2008;98(7):1210-1216.

[10] Mehta VK, Cho C, Ford JM, et al. Phase II trial of preoperative 3D conformal radiotherapy, protracted venous infusion 5fluorouracil, and weekly CPT-11, followed by surgery for ultrasound-staged T3 rectal cancer. Int J Radiat Oncol Biol Phys 2003;55(1):132-137.

[11] Mohiuddin M, Winter K, Mitchell E, et al. Randomized phase II study of neoadjuvant combined-modality chemoradiation for distal rectal cancer: Radiation Therapy Oncology Group Trial 0012. J Clin Oncol 2006;24(4):650-655.

[12] Navarro M, Dotor E, Rivera F, et al. A phase II study of preoperative radiotherapy and concomitant weekly irinotecan in combination with protracted venous infusion 5fluorouracil, for resectable locally advanced rectal cancer. Int J Radiat Oncol Biol Phys 2006;66(1):201-205.

[13] Willeke F, Horisberger K, Kraus-Tiefenbacher U, et al. A phase II study of capecitabine and irinotecan in combination with concurrent pelvic radiotherapy (CapIri-RT) as neoadjuvant treatment of locally advanced rectal cancer. $\mathrm{Br} J$ Cancer 2007;96(6):912-917.

[14] Gollins S, Myint AS, Haylock B, et al. Preoperative chemoradiotherapy using concurrent capecitabine and irinotecan in magnetic resonance imaging-defined locally advanced rectal cancer: impact on long-term clinical outcomes. J Clin Oncol 2011;29(8):1042-1049.

[15] Yoon WS, Chae YS, Hong J, Park YK. Antitumor therapeutic effects of a genetically engineered Salmonella typhimurium harboring TNF-alpha in mice. Appl Microbiol Biotechnol 2011;89(6):1807-1819.

[16] Sato T, Ozawa $H$, Hatate $K$, et al. A phase II trial of neoadjuvant preoperative chemoradiotherapy with S-1 plus irinotecan and radiation in patients with locally advanced rectal cancer: clinical feasibility and response rate. Int J Radiat Oncol Biol Phys 2011;79(3):677-683.

[17] Jung M, Shin SJ, Lim S, et al. A randomized phase II study of neoadjuvant chemoradiotherapy with 5-FU/leucovorin or irinotecan/S1 in patients with locally advanced rectal cancer. J Clin Oncol 2013;31(4S):511.

[18] Cai G, Zhu J, Palmer JD, et al. CAPIRI-IMRT: a phase II study of concurrent capecitabine and irinotecan with intensitymodulated radiation therapy for the treatment of recurrent rectal cancer. Radiat Oncol 2015;10:57.

[19] Mohiuddin M, Paulus R, Mitchell E, et al. Neoadjuvant chemoradiation for distal rectal cancer: 5-year updated results of a randomized phase 2 study of neoadjuvant combined modality chemoradiation for distal rectal cancer. Int J Radiat Oncol Biol Phys 2013;86(3):523-528.

[20] Nakamura T, Yamashita $K$, Sato T, Ema A, Naito M, Watanabe M. Neoadjuvant chemoradiation therapy using concurrent S-1 and irinotecan in rectal cancer: impact on long-term clinical outcomes and prognostic factors. Int J Radiat Oncol Biol Phys 2014;89(3):547-555.

[21] UK Clinical Research Network. UK Clinical Research Network Portfolio Database: Aristotle 2015. http://public. ukcrn.org.uk/search/StudyDetail.aspx?StudyID =7890 [accessed 23.07.15].

[22] Hill EJ, Nicolay NH, Middleton MR, Sharma RA. Oxaliplatin as a radiosensitiser for upper and lower gastrointestinal tract malignancies: what have we learned from a decade of translational research? Crit Rev Oncol Hematol 2012;83(3):353-387.

[23] Zhu AX, Willett CG. Chemotherapeutic and biologic agents as radiosensitizers in rectal cancer. Semin Radiat Oncol 2003;13(4):454-468.

[24] Rutten H, Sebag-Montefiore D, Glynne-Jones R, et al. Capecitabine, oxaliplatin, radiotherapy, and excision (CORE) in patients with MRI-defined locally advanced rectal adenocarcinoma: results of an international multicenter phase II study. J Clin Oncol 2006;24(18S):3528.

[25] Carlomagno C, Farella A, Bucci L, et al. Neo-adjuvant treatment of rectal cancer with capecitabine and oxaliplatin in combination with radiotherapy: a phase II study. Ann Oncol 2009;20(5):906-912.

[26] Koeberle D, Burkhard R, von Moos R, et al. Phase II study of capecitabine and oxaliplatin given prior to and concurrently with preoperative pelvic radiotherapy in patients with 
locally advanced rectal cancer. Br J Cancer 2008;98(7): 1204-1209.

[27] Rosenthal DI, Catalano PJ, Haller DG, et al. Phase I study of preoperative radiation therapy with concurrent infusional 5fluorouracil and oxaliplatin followed by surgery and postoperative 5-fluorouracil plus leucovorin for T3/T4 rectal adenocarcinoma: ECOG E1297. Int J Radiat Oncol Biol Phys 2008;72(1):108-113.

[28] Gerard JP, Azria D, Gourgou-Bourgade S, et al. Comparison of two neoadjuvant chemoradiotherapy regimens for locally advanced rectal cancer: results of the phase III trial ACCORD 12/0405-Prodige 2. J Clin Oncol 2010;28(10): $1638-1644$.

[29] Gerard JP, Azria D, Gourgou-Bourgade S, et al. Clinical outcome of the ACCORD 12/0405 PRODIGE 2 randomized trial in rectal cancer. $J$ Clin Oncol 2012;30(36):4558-4565.

[30] Aschele C, Cionini L, Lonardi S, et al. Primary tumor response to preoperative chemoradiation with or without oxaliplatin in locally advanced rectal cancer: pathologic results of the STAR-01 randomized phase III trial. J Clin Oncol 2011;29(20):2773-2780.

[31] Rodel C, Liersch T, Becker $\mathrm{H}$, et al. Preoperative chemoradiotherapy and postoperative chemotherapy with fluorouracil and oxaliplatin versus fluorouracil alone in locally advanced rectal cancer: initial results of the German CAO/ ARO/AIO-04 randomised phase 3 trial. Lancet Oncol 2012;13(7):679-687.

[32] Rödel C, Graeven U, Fietkau R, et al. Oxaliplatin added to fluorouracil-based preoperative chemoradiotherapy and postoperative chemotherapy of locally advanced rectal cancer (the German CAO/ARO/AIO-04 study): final results of the multicentre, open-label, randomised, phase 3 trial. Lancet Oncol 2015;16(8):979-989.

[33] Schmoll HJ, Haustermans K, Price T, et al. Preoperative chemoradiotherapy and postoperative chemotherapy with capecitabine and oxaliplatin versus capecitabine alone in locally advanced rectal cancer: response to the local treatment after chemoradiation and surgery as secondary endpoint. Eur J Cancer 2013;49. S482-S482.

[34] Schmoll HJ, Haustermans K, Price TJ, et al. Preoperative chemoradiotherapy and postoperative chemotherapy with capecitabine \pm oxaliplatin in locally advanced rectal cancer: interim analysis for disease-free survival of PETACC 6. Ann Oncol 2014;25(4S):iv170-iv171.

[35] O'Connell MJ, Colangelo LH, Beart RW, et al. Capecitabine and oxaliplatin in the preoperative multimodality treatment of rectal cancer: surgical end points from National Surgical Adjuvant Breast and Bowel Project trial R-04. J Clin Oncol 2014;32(18):1927-1934.

[36] Allegra CJ, Yothers G, O'Connell MJ, et al. Final results from NSABP protocol R-04: Neoadjuvant chemoradiation (RT) comparing continuous infusion (CIV) 5-FU with capecitabine (Cape) with or without oxaliplatin (Ox) in patients with stage II and III rectal cancer. ASCO Meeting Abstracts 2014;32(15S):3603.

[37] An X, Lin X, Wang FH, et al. Short term results of neoadjuvant chemoradiotherapy with fluoropyrimidine alone or in combination with oxaliplatin in locally advanced rectal cancer: a meta analysis. Eur J Cancer 2013;49(4):843-851.

[38] Gerard JP, Azria D, Sophie GB, Conroy T, Bedenne L. Clinical results at 3 years of the ACCORD 12 randomized trial in rectal cancer. J Clin Oncol 2012;30(4S):389.

[39] Wong SJ, Winter K, Meropol NJ, et al. Radiation Therapy Oncology Group 0247: a randomized phase II study of neoadjuvant capecitabine and irinotecan or capecitabine and oxaliplatin with concurrent radiotherapy for patients with locally advanced rectal cancer. Int J Radiat Oncol Biol Phys 2012;82(4):1367-1375.

[40] Lee CG, Heijn M, di Tomaso E, et al. Anti-vascular endothelial growth factor treatment augments tumor radiation response under normoxic or hypoxic conditions. Cancer Res 2000;60(19):5565-5570.

[41] Willett CG, Boucher Y, di Tomaso E, et al. Direct evidence that the VEGF-specific antibody bevacizumab has antivascular effects in human rectal cancer. Nat Med 2004;10(2):145-147.

[42] Kerbel RS. Tumor angiogenesis. New Engl J Med 2008;358(19):2039-2049.

[43] Fornaro L, Caparello C, Vivaldi C, et al. Bevacizumab in the pre-operative treatment of locally advanced rectal cancer: a systematic review. World J Gastroenterol 2014;20(20): 6081-6091.

[44] Torino F, Sarmiento R, Gasparini G. The contribution of targeted therapy to the neoadjuvant chemoradiation of rectal cancer. Crit Rev Oncol Hematol 2013;87(3):283-305.

[45] Marijnen CA, Rutten H, de Wilt H, et al. Preoperative chemoradiotherapy regimen with capecitabine and bevacizumab in locally advanced rectal cancer: a feasibility study of the Dutch Colorectal Cancer Group (DCCG). J Clin Oncol 2008;26(15S):15040.

[46] Willett CG, Duda DG, di Tomaso E, et al. Efficacy, safety, and biomarkers of neoadjuvant bevacizumab, radiation therapy, and fluorouracil in rectal cancer: a multidisciplinary phase II study. J Clin Oncol 2009;27(18):3020-3026.

[47] Willett CG, Duda DG, Ancukiewicz M, et al. A safety and survival analysis of neoadjuvant bevacizumab with standard chemoradiation in a phase I/II study compared with standard chemoradiation in locally advanced rectal cancer. Oncologist 2010;15(8):845-851.

[48] Crane CH, Eng C, Feig BW, et al. Phase II trial of neoadjuvant bevacizumab, capecitabine, and radiotherapy for locally advanced rectal cancer. Int J Radiat Oncol Biol Phys 2010;76(3):824-830.

[49] Nogue M, Salud A, Vicente P, et al. Addition of bevacizumab to XELOX induction therapy plus concomitant capecitabinebased chemoradiotherapy in magnetic resonance imagingdefined poor-prognosis locally advanced rectal cancer: the AVACROSS study. Oncologist 2011;16(5):614-620.

[50] Spigel DR, Bendell JC, McCleod M, et al. Phase II study of bevacizumab and chemoradiation in the preoperative or adjuvant treatment of patients with stage II/III rectal cancer. Clin Colorectal Cancer 2012;11(1):45-52.

[51] Velenik V, Ocvirk J, Music M, et al. Neoadjuvant capecitabine, radiotherapy, and bevacizumab (CRAB) in locally advanced rectal cancer: results of an open-label phase II study. Radiat Oncol 2011;6:105.

[52] Velenik V, Ocvirk J, Music M, et al. CRAB trial: Long-term results from a prospective phase II study evaluating neoadjuvant capecitabine, radiotherapy (RT) and revacizumab in locally advanced rectal cancer. Eur J Cancer 2013;49:S506.

[53] Resch G, De Vries A, Oefner D, et al. Preoperative treatment with capecitabine, bevacizumab and radiotherapy for primary locally advanced rectal cancer - a two stage phase II clinical trial. Radiother Oncol 2012;102(1):10-13.

[54] Gasparini G, Torino F, Ueno T, et al. A phase II study of neoadjuvant bevacizumab plus capecitabine and concomitant radiotherapy in patients with locally advanced rectal cancer. Angiogenesis 2012;15(1):141-150.

[55] Salazar R, Capdevila J, Laquente B, et al. A randomized phase II study of capecitabine-based chemoradiation with or without 
bevacizumab in resectable locally advanced rectal cancer: clinical and biological features. BMC Cancer 2015;15:60.

[56] Garcia M, Martinez-Villacampa M, Santos C, et al. Phase Il study of preoperative bevacizumab, capecitabine and radiotherapy for resectable locally-advanced rectal cancer. BMC Cancer 2015;15:59.

[57] Czito BG, Bendell JC, Willett CG, et al. Bevacizumab, oxaliplatin, and capecitabine with radiation therapy in rectal cancer: phase I trial results. Int J Radiat Oncol Biol Phys 2007;68(2):472-478.

[58] Dipetrillo T, Pricolo V, Lagares-Garcia J, et al. Neoadjuvant bevacizumab, oxaliplatin, 5-fluorouracil, and radiation for rectal cancer. Int J Radiat Oncol Biol Phys 2012;82(1): 124-129.

[59] Kennecke H, Berry S, Wong R, et al. Pre-operative bevacizumab, capecitabine, oxaliplatin and radiation among patients with locally advanced or low rectal cancer: a phase II trial. Eur J Cancer 2012;48(1):37-45.

[60] Dellas K, Hohler T, Reese T, et al. Phase II trial of preoperative radiochemotherapy with concurrent bevacizumab, capecitabine and oxaliplatin in patients with locally advanced rectal cancer. Radiat Oncol 2013;8:90.

[61] Landry JC, Feng Y, Cohen SJ, et al. Phase 2 study of preoperative radiation with concurrent capecitabine, oxaliplatin, and bevacizumab followed by surgery and postoperative 5fluorouracil, leucovorin, oxaliplatin (FOLFOX), and bevacizumab in patients with locally advanced rectal cancer: ECOG 3204. Cancer 2013;119(8):1521-1527.

[62] Landry JC, Feng Y, Prabhu RS, et al. Phase II trial of preoperative radiation with concurrent capecitabine, oxaliplatin, and bevacizumab followed by surgery and postoperative 5-fluorouracil, leucovorin, oxaliplatin (FOLFOX), and bevacizumab in patients with locally advanced rectal cancer: 5-year clinical outcomes ECOGACRIN Cancer Research Group E3204. Oncologist 2015;20(6):615-616.

[63] Das P, Eng C, Rodriguez-Bigas MA, et al. Preoperative radiation therapy with concurrent capecitabine, bevacizumab, and erlotinib for rectal cancer: a phase 1 trial. Int $J$ Radiat Oncol Biol Phys 2014;88(2):301-305.

[64] Blaszkowsky LS, Ryan DP, Szymonifka J, et al. Phase I/II study of neoadjuvant bevacizumab, erlotinib and 5-fluorouracil with concurrent external beam radiation therapy in locally advanced rectal cancer. Ann Oncol 2014;25(1):121-126.

[65] Koukourakis MI, Giatromanolaki A, Tsoutsou P, et al. Bevacizumab, capecitabine, amifostine, and preoperative hypofractionated accelerated radiotherapy (HypoArc) for rectal cancer: a phase II study. Int J Radiat Oncol Biol Phys 2011;80(2):492-498.

[66] Koukourakis MI, Giatromanolaki A, Sheldon H, et al. Phase I/ II trial of bevacizumab and radiotherapy for locally advanced inoperable colorectal cancer: vasculature-independent radiosensitizing effect of bevacizumab. Clin Cancer Res 2009;15(22):7069-7076.

[67] Nyati MK, Morgan MA, Feng FY, Lawrence TS. Integration of EGFR inhibitors with radiochemotherapy. Nat Rev Cancer 2006;6(11):876-885.

[68] Bonner JA, Harari PM, Giralt J, et al. Radiotherapy plus cetuximab for squamous-cell carcinoma of the head and neck. N Engl J Med 2006;354(6):567-578.

[69] Chung KY, Minsky B, Schrag D, et al. Phase I trial of preoperative cetuximab with concurrent continuous infusion 5fluorouracil and pelvic radiation in patients with localregionally advanced rectal cancer. ASCO Meeting Abstracts 2006;24(18S):3560.
[70] Machiels J-P, Sempoux C, Scalliet P, et al. Phase I/II study of preoperative cetuximab, capecitabine, and external beam radiotherapy in patients with rectal cancer. Ann Oncol 2007; 18(4):738-744.

[71] Semrau R, Kocher M, Vallbohmer D, Hoelscher A. Combination of 5FU-based chemoradiation with EGFR inhibitor cetuximab and effect on PCR rate in patients undergoing neoadjuvant treatment in advanced rectal cancer. J Clin Oncol 2014;32(3S):623.

[72] Bertolini F, Chiara S, Bengala C, et al. Neoadjuvant treatment with single-agent cetuximab followed by 5-FU, cetuximab, and pelvic radiotherapy: a phase II study in locally advanced rectal cancer. Int $J$ Radiat Oncol Biol Phys 2009;73(2):466-472.

[73] Velenik V, Ocvirk J, Oblak I, Anderluh F. A phase II study of cetuximab, capecitabine and radiotherapy in neoadjuvant treatment of patients with locally advanced resectable rectal cancer. Eur J Surg Oncol 2010;36(3):244-250.

[74] Dewdney A, Cunningham D, Tabernero J, et al. Multicenter randomized phase II clinical trial comparing neoadjuvant oxaliplatin, capecitabine, and preoperative radiotherapy with or without cetuximab followed by total mesorectal excision in patients with high-risk rectal cancer (EXPERT-C). J Clin Oncol 2012;30(14):1620-1627.

[75] Sun PL, Li B, Ye QF. Effect of neoadjuvant cetuximab, capecitabine, and radiotherapy for locally advanced rectal cancer: results of a phase II study. Int $J$ Colorectal Dis 2012;27(10):1325-1332.

[76] McCollum AD, Kocs DM, Chadha P, et al. Randomized phase II trial of preoperative chemoradiotherapy with or without cetuximab in locally advanced rectal adenocarcinoma. J Clin Oncol 2014;32(3S):537.

[77] Eisterer W, De Vries A, Ofner D, et al. Preoperative treatment with capecitabine, cetuximab and radiotherapy for primary locally advanced rectal cancer - a phase II clinical trial. Anticancer Res 2014;34(11):6767-6773.

[78] Hofheinz RD, Horisberger K, Woernle C, et al. Phase I trial of cetuximab in combination with capecitabine, weekly irinotecan, and radiotherapy as neoadjuvant therapy for rectal cancer. Int J Radiat Oncol Biol Phys 2006;66(5): 1384-1390.

[79] Horisberger K, Treschl A, Mai S, et al. Cetuximab in combination with capecitabine, irinotecan, and radiotherapy for patients with locally advanced rectal cancer: results of a phase II MARGIT trial. Int J Radiat Oncol Biol Phys 2009;74(5):1487-1493.

[80] Kim SY, Hong YS, Kim DY, et al. Preoperative chemoradiation with cetuximab, irinotecan, and capecitabine in patients with locally advanced resectable rectal cancer: a multicenter Phase II study. Int J Radiat Oncol Biol Phys 2011;81(3): $677-683$.

[81] Gollins S, Myint AS, Saunders MP, et al. Excite: a phase II trial of preoperative cetuximab, irinotecan and capecitabine plus radiotherapy (RT) in mri-defined locally advanced rectal cancer (LARC). Ann Oncol 2012;23:ix206-ix207.

[82] Gollins S, Quirke P, Myint S, et al. Pretreatment and postresection epidermal growth factor receptor (EGFR) pathway mutations in a prospective phase II trial (EXCITE) of preoperative cetuximab-containing chemoradiation (CRT) in locally advanced rectal cancer (LARC). J Clin Oncol 2014;32(3S):458.

[83] Cabebe EC, Kuo T, Koong A, et al. Phase I trial of preoperative cetuximab in combination with oxaliplatin, capecitabine, and radiation therapy for locally advanced rectal cancer. J Clin Oncol 2008;26(15S May 20 Supplement):15019. 
[84] Rodel C, Arnold D, Hipp M, et al. Phase I-II trial of cetuximab, capecitabine, oxaliplatin, and radiotherapy as preoperative treatment in rectal cancer. Int J Radiat Oncol Biol Phys 2008;70(4):1081-1086.

[85] Azria D, Gourqou-Bourqade S, Kaminsky MC, et al. Combined strategy of preoperative radiochemotherapy (50 Gy + capecitabine + oxaliplatin + cetuximab), surgery, and postoperative chemotherapy (folfox $4+$ cetuximab) in locally advanced rectal cancer with synchronous resectable metastases: Interim results of the french multicentric phase II trial (ACCORD 14/0604). Radiother Oncol 2010;96(1S):S277.

[86] Elvira G, Torrecillas L, Cervantes G, Erzao Valle Solis AA. Phase II study of bevacizumab and cetuximab as neoadjuvant treatment in locally advanced rectal cancer: a preliminary security report. J Clin Oncol 2011;29(4S):627.

[87] Czito BG, Willett CG, Bendell JC, et al. Increased toxicity with gefitinib, capecitabine, and radiation therapy in pancreatic and rectal cancer: phase I trial results. J Clin Oncol 2006;24(4):656-662.

[88] Valentini V, De Paoli A, Gambacorta MA, et al. Infusional 5fluorouracil and ZD1839 (Gefitinib-Iressa) in combination with preoperative radiotherapy in patients with locally advanced rectal cancer: a phase I and II trial (1839IL/0092). Int J Radiat Oncol Biol Phys 2008;72(3):644-649.

[89] Mardjuadi FI, Carrasco J, Coche JC, et al. Panitumumab as a radiosensitizing agent in KRAS wild-type locally advanced rectal cancer. Target Oncol 2015;10(3):375-383.

[90] Jin T, Zhu Y, Luo JL, et al. Prospective phase II trial of nimotuzumab in combination with radiotherapy and concurrent capecitabine in locally advanced rectal cancer. Int J Colorectal Dis 2015;30(3):337-345.

[91] Hong YS, Kim DY, Lim SB, et al. Preoperative chemoradiation with irinotecan and capecitabine in patients with locally advanced resectable rectal cancer: long-term results of a Phase II study. Int J Radiat Oncol Biol Phys 2011;79(4):1171-1178.

[92] Kim SY, Shim EK, Yeo HY, et al. KRAS mutation status and clinical outcome of preoperative chemoradiation with cetuximab in locally advanced rectal cancer: a pooled analysis of 2 phase II trials. Int J Radiat Oncol Biol Phys 2013;85(1):201-207.

[93] Kripp M, Horisberger K, Mai S, et al. Does the addition of cetuximab to radiochemotherapy improve outcome of patients with locally advanced rectal cancer? Long-term results from phase II trials. Gastroenterol Res Pract 2015;2015:8.

[94] Hofheinz RD, von Gerstenberg-Helldorf B, Wenz F, et al. Phase I trial of capecitabine and weekly irinotecan in combination with radiotherapy for neoadjuvant therapy of rectal cancer. J Clin Oncol 2005;23(7):1350-1357.

[95] Sclafani F, Peckitt C, Cunningham D, et al. Panex: a pooled analysis of EXPERT and EXPERT-C, two trials of neoadjuvant chemotherapy (NACT) and chemoradiotherapy (CRT) in high-risk locally advanced rectal cancer (LARC). J Clin Oncol 2014;32(15S):3575.

[96] Powell C, Mikropoulos C, Kaye SB, et al. Pre-clinical and clinical evaluation of PARP inhibitors as tumour-specific radiosensitisers. Cancer Treat Rev 2010;36(7):566-575.

[97] Vilar E, Gruber SB. Microsatellite instability in colorectal cancer-the stable evidence. Nat Rev Clin Oncol 2010;7(3):153-162.

[98] Czito BG, Mulcahy MF, Deming DA, et al. The safety and tolerability of veliparib (V) plus capecitabine (C) and radiation (RT) in subjects with locally advanced rectal cancer (LARC): results of a phase $1 \mathrm{~b}$ study. J Clin Oncol 2015;33(3):579.
[99] Groselj B, Sharma NL, Hamdy FC, Kerr M, Kiltie AE. Histone deacetylase inhibitors as radiosensitisers: effects on DNA damage signalling and repair. Br J Cancer 2013;108(4): $748-754$.

[100] Ree AH, Dueland S, Folkvord S, et al. Vorinostat, a histone deacetylase inhibitor, combined with pelvic palliative radiotherapy for gastrointestinal carcinoma: the Pelvic Radiation and Vorinostat (PRAVO) phase 1 study. Lancet Oncol 2010;11(5):459-464.

[101] Hill E, Franklin J, Enescu M, et al. Phase 0-I clinical trial of nelfinavir with hypofractionated radiotherapy for rectal cancer: clinical safety and changes in tumour perfusion observed. National Cancer Research Institute Conference 2012. RCR26.

[102] Buijsen J, Lammering G, Jansen RL, et al. Phase I trial of the combination of the Akt inhibitor nelfinavir and chemoradiation for locally advanced rectal cancer. Radiother Oncol 2013;107(2):184-188.

[103] Marti-Marti FE, Miles L, Allen J, et al. A novel trial design for rectal cancer: the Dreamtherapytrial (Dual Rectal Angiogenesis Mek Inhibition Radiotherapy). Ann Oncol 2011;22(5S):130.

[104] Von Moos R, Koeberle D, Schacher S, et al. Neoadjuvant radiotherapy (RT) combined with capecitabine (Cape) and sorafenib (Sor) in patients (pts) with locally advanced, k-rasmutated rectal cancer (LARC): a phase I/II trial SAKK 41/08. J Clin Oncol 2014;32(15S):3531.

[105] Malik I, Hussein F, Bush D, et al. A phase I study of capecitabine, irinotecan, celecoxib, and radiation as neoadjuvant therapy of patients with locally advanced rectal cancer. Am J Clin Oncol 2010;33(3):242-245.

[106] O'Neil BH, Raftery L, Calvo BF, et al. A phase I study of bortezomib in combination with standard 5-fluorouracil and external-beam radiation therapy for the treatment of locally advanced or metastatic rectal cancer. Clin Colorectal Cancer 2010;9(2):119-125.

[107] Citrin D, Camphausen K, Wood BJ, et al. A pilot feasibility study of TNFerade (TM) biologic with capecitabine and radiation therapy followed by surgical resection for the treatment of rectal cancer. Oncology 2010;79(5-6):382-388.

[108] Illum H, Wang DH, Dowell JE, et al. Phase I dose escalation trial of nitroglycerin in addition to 5-fluorouracil and radiation therapy for neoadjuvant treatment of operable rectal cancer. Surgery 2015;158(2):460-465.

[109] The Cancer Genome Atlas Network. Comprehensive molecular characterization of human colon and rectal cancer. $\mathrm{Na}$ ture 2012;487(7407):330-337.

[110] Stegeman H, Span PN, Kaanders JH, Bussink J. Improving chemoradiation efficacy by PI3-K/AKT inhibition. Cancer Treat Rev 2014;40(10):1182-1191.

[111] Pore N, Gupta AK, Cerniglia GJ, et al. Nelfinavir downregulates hypoxia-inducible factor $1 \alpha$ and VEGF expression and increases tumor oxygenation: implications for radiotherapy. Cancer Res 2006;66(18):9252-9259.

[112] Brunner TB, Geiger M, Grabenbauer GG, et al. Phase I trial of the human immunodeficiency virus protease inhibitor nelfinavir and chemoradiation for locally advanced pancreatic cancer. J Clin Oncol 2008;26(16):2699-2706.

[113] Rengan R, Mick R, Pryma D, et al. A phase I trial of the HIV protease inhibitor nelfinavir with concurrent chemoradiotherapy for unresectable stage IIIA/IIIB non-small cell lung cancer: a report of toxicities and clinical response. J Thoracic Oncol 2012;7(4):709-715.

[114] Russo SM, Tepper JE, Baldwin Jr AS, et al. Enhancement of radiosensitivity by proteasome inhibition: implications for a 
role of NF-kappaB. Int $J$ Radiat Oncol Biol Phys 2001;50(1):183-193.

[115] Edelman MJ, Burrows W, Krasna MJ, Bedor M, Smith R, Suntharalingam M. Phase I trial of carboplatin/paclitaxel/ bortezomib and concurrent radiotherapy followed by surgical resection in stage III non-small cell lung cancer. Lung Cancer 2010;68(1):84-88.

[116] Demaria S, Golden EB, Formenti SC. Role of local radiation therapy in cancer immunotherapy. JAMA Oncol 2015:E1-E8.

[117] Illidge $T$. Turning radiotherapy into an effective systemic anti-cancer treatment in combination with immunotherapy. Clin Oncol 2015;27(12):696-699.

[118] Dovedi SJ, Adlard AL, Lipowska-Bhalla G, et al. Acquired resistance to fractionated radiotherapy can be overcome by concurrent PD-L1 blockade. Cancer Res 2014;74(19):5458-5468.

[119] Schumacher TN, Schreiber RD. Neoantigens in cancer immunotherapy. Science 2015;348(6230):69-74.

[120] Glynne-Jones R, Hadaki M, Harrison M. The status of targeted agents in the setting of neoadjuvant radiation therapy in locally advanced rectal cancers. J Gastrointest Oncol 2013;4(3):264-284.

[121] Bratland A, Dueland S, Hollywood D, Flatmark K, Ree AH. Gastrointestinal toxicity of vorinostat: reanalysis of phase 1 study results with emphasis on dose-volume effects of pelvic radiotherapy. Radiat Oncol 2011;6:33.
[122] Cubillo A, Hernando-Requejo O, Garcia-Garcia E, et al. A prospective pilot study of target-guided personalized chemotherapy with intensity-modulated radiotherapy in patients with early rectal cancer. Am J Clin Oncol 2014;37(2):117-121.

[123] Medical Research Council Clinical Trials Unit. FOCUS 42014. http://www.focus4trial.org/ [accessed 03.08.15].

[124] de Campos-Lobato LF, Stocchi L, da Luz Moreira A, et al. Pathologic complete response after neoadjuvant treatment for rectal cancer decreases distant recurrence and could eradicate local recurrence. Ann Surg Oncol 2011;18(6): 1590-1598.

[125] Maas M, Nelemans PJ, Valentini V, et al. Long-term outcome in patients with a pathological complete response after chemoradiation for rectal cancer: a pooled analysis of individual patient data. Lancet Oncol 2010;11(9):835-844.

[126] Stuart J Wong, Jennifer Moughan, Neal J Meropol, et al. Efficacy Endpoints of Radiation Therapy Group Protocol 0247: A Randomized, Phase 2 Study of Neoadjuvant Radiation Therapy Plus Concurrent Capecitabine and Irinotecan or Capecitabine and Oxaliplatin for Patients With Locally Advanced Rectal Cancer. International Journal of Radiation Oncology Biology Physics 2015;91(1):116-123. http:// dx.doi.org/10.1016/j.ijrobp.2014.09.031. 